\title{
The Chern character of semifinite spectral triples
}

\author{
Alan L. Carey, John Phillips, Adam Rennie, and Fyodor A. Sukochev*
}

\begin{abstract}
In previous work we generalised both the odd and even local index formula of Connes and Moscovici to the case of spectral triples for a $*$-subalgebra $\mathcal{A}$ of a general semifinite von Neumann algebra. Our proofs are novel even in the setting of the original theorem and rely on the introduction of a function valued cocycle (called the resolvent cocycle) which is 'almost' a $(b, B)$-cocycle in the cyclic cohomology of $\mathcal{A}$. In this paper we show that this resolvent cocycle 'almost' represents the Chern character and assuming analytic continuation properties for zeta functions we show that the associated residue cocycle, which appears in our statement of the local index theorem does represent the Chern character.
\end{abstract}

Mathematics Subject Classification (2000). Primary: 19K56, 46L80; Secondary: 58B30, 46L87.

Keywords. Von Neumann algebra, Fredholm module, cyclic cohomology, Chern character, spectral flow.

\section{Introduction}

The local index theorem in noncommutative geometry is due to Connes and Moscovici [CoM]. The main consequence of the theorem is a formula for the Chern character of an unbounded Fredholm module (or spectral triple) in terms of a 'residue cocycle' in the $(b, B)$ bicomplex. This residue cocycle is a sum of residues of certain zeta functions. There have been two new proofs of this formula discovered recently, by Higson $[\mathrm{H}]$ and by the present authors [CPRS2], [CPRS3]. The main new feature of these proofs is that the starting point for the Connes-Moscovici argument (the JLO cocycle [JLO], [Co1]) is replaced by a different cocycle derived from the resolvent expansion in perturbation theory. These new proofs have some conceptual and technical advantages over the earlier proof. In particular [CPRS2], [CPRS3] enable the local index theorem to be extended to the case where one has a spectral triple 'inside' a general semifinite von Neumann algebra $\mathcal{N}$. This extension enabled us to

\footnotetext{
*All authors were supported by grants from ARC (Australia) and NSERC (Canada), in addition the third named author acknowledges a University of Newcastle early career researcher grant and support from the SNF, Denmark.
} 
encompass examples of operators that are not Fredholm in the ordinary sense but are Breuer-Fredholm (see [BeF], [CP1], [Sh], [L], [M], [BCPRSW]).

The semifinite version of the local index formula is proved using a holomorphic function valued 'resolvent cocycle' in the $(b, B)$ bicomplex. It has some similarities to the 'improper cocycle' of $[\mathrm{H}]$ which is also function valued but has very different holomorphy properties to our resolvent cocycle. In the last section of this paper we clarify the relationship of our work with $[\mathrm{H}]$.

Currently absent from earlier work is a detailed exposition of the properties of our resolvent cocycle which would put it on the same footing as the JLO coycle. To that end we provide a uniform exposition of both the odd and even forms of the cocycle that were introduced separately in each of [CPRS2] (for the odd case) and [CPRS3] (for the even case). Then we give a proof that our resolvent cocycle represents (in a suitable sense) the 'semifinite Chern character in cyclic cohomology'. From this we derive some important consequences which we will summarise later in the introduction. We note that this paper also adds to the general understanding of semifinite noncommutative geometry started in [CP1], [BeF].

The hypotheses for our proof of the local index formula are weaker than those of $[\mathrm{CoM}]$. We assume that we have a finitely summable spectral triple $(\mathcal{A}, \mathscr{H}, \mathcal{D})$ with spectral dimension $q$ (that is $\left(1+\mathscr{D}^{2}\right)^{-n / 2}$ is trace class for all $n>q$ and $q$ is the least positive real number for which this is true). This suffices to verify that the individual functionals that make up the resolvent cocycle are continuous in an appropriate sense with values in functions defined and holomorphic in a certain half-plane. We obtain a cocycle in the finite $(b, B)$ complex by considering the resolvent cocycle modulo those functions holomorphic in a half-plane containing the critical point $r=(1-q) / 2$ (where $a$ priori the terms in the resolvent cocycle may have a singularity and where we take residues to obtain our version of the Connes-Moscovici residue cocycle). We remark, but do not prove here, that the resolvent cocycle is not entire but at no point do we need infinitely many terms in our expression for this cocycle. We use the notation $\left(\phi_{m}^{r}\right), m=0,1,2, \ldots$ to denote the components of the resolvent cocycle in the $(b, B)$ complex ( $m$ odd in the odd case, even in the even case).

In our proof of the local index formula in [CPRS2], [CPRS3] we showed that if the spectral triple has a property we termed 'isolated spectral dimension', which is weaker than the assumption of 'discrete dimension spectrum' used in [CoM], then we can evaluate the resolvent cocycle term-by-term by taking residues at the critical point $r=(1-q) / 2$. The resulting formula gives an index of a Breuer-Fredholm operator expressed in terms of the residues of zeta functions at the critical point and these residue functionals assemble to give a version of the Connes-Moscovici residue cocycle. Our formula is not identical with that of [CoM] because we need to deal, in the von Neumann context, with the problem that zero may be in the continuous spectrum of $\mathcal{D}$.

In this paper there are two main theorems. The first proves that the resolvent cocycle 'almost' represents $(r-(1-q) / 2)^{-1}$ times the Chern character Ch of our 
semifinite spectral triples in the sense that they are cohomologous modulo functions which are holomorphic at $r=(1-q) / 2$. We use the notation

$$
\left(\phi_{m}^{r}\right)_{m=0}^{M} \sim\left(\frac{1}{r-(1-q) / 2} \mathrm{Ch}\right)
$$

to represent this fact. Our second main theorem proves that our residue cocycle exactly represents the Chern character.

One consequence of these results is to provide an alternative proof of the semifinite local index formula in the case of odd spectral triples. This is because in [CPRS2], in order to show that the residue cocycle we obtain from the resolvent cocycle calculates the appropriate Breuer-Fredholm index we had to start with the spectral flow formula of [CP2]. This spectral flow formula is quite difficult to prove in the case of general semifinite spectral triples and it is desirable to find a more direct argument. Our second main theorem does this as we may now use the known fact [Co1] that the Chern character is an index cocycle to bypass the formula of [CP2]. (We note that, although the argument in [Co1] that shows that the Chern character calculates a Fredholm index, is formulated only in the standard case of spectral triples with $\mathcal{N}$ being all bounded operators on a separable Hilbert space, the results of [CPRS3] enable one to see that the arguments of [Co1] go through without essential change for semifinite spectral triples.)

Benefits of our discussion include the exposition of technical details for the semifinite version of the transgression to the Chern character which differ from the type I case $[\mathrm{H}]$ (the latter relies on Lemme 8 of the Comptes Rendus note [CM1]). We also give an account of the relationship between the resolvent cocycle and Higson's 'improper cocycle'. In fact we obtain from our cocycle a renormalised version of this 'improper cocycle' (which we call the reduced resolvent cocycle). Finally we obtain analytic continuation results for the resolvent cocycle evaluated on Hochschild cycles (of top degree) and on $(b, B)$ cycles. These results do not require assumptions on analytic continuation properties of individual zeta functions.

The exposition is organised as follows. We put preliminary material on semifinite spectral triples, needed for the main results, in Section 2 including a brief outline of the pseudodifferential calculus. Those parts of cyclic theory we need are introduced in Section 3. Those expert in these matters can move straight to Section 4 where we introduce the various cocycles which arose in our new proof of the local index formula. Then we state our main theorem on the residue cocycle. The proof is in Section 5 where we first prove that in both the even and odd cases, the resolvent cocycle 'almost' represents the Chern character and we show that this implies that the residue cocycle is in the class of the Chern character. Section 6 derives some corollaries including the relationship to Higson's point of view. 


\section{Definitions and background}

2.1. Semifinite spectral triples. We begin with some semifinite versions of standard definitions and results. Let $\tau$ be a fixed faithful, normal, semifinite trace on the von Neumann algebra $\mathcal{N}$. Let $\mathcal{K}_{\mathcal{N}}$ be the $\tau$-compact operators in $\mathcal{N}$ (that is the norm closed ideal generated by the projections $E \in \mathcal{N}$ with $\tau(E)<\infty)$.

Definition 2.1. A semifinite spectral triple $(\mathcal{A}, \mathscr{H}, \mathscr{D})$ is given by a Hilbert space $\mathscr{H}$, a $*$-algebra $\mathcal{A} \subset \mathcal{N}$ where $(\mathcal{N}, \tau)$ is a semifinite von Neumann algebra with trace $\tau$ acting on $\mathscr{H}$, and a densely defined unbounded self-adjoint operator $\mathscr{D}$ affiliated to $\mathcal{N}$ such that $[\mathscr{D}, a]$ is densely defined and extends to a bounded operator in $\mathcal{N}$ for all $a \in \mathcal{A}$ and $(\lambda-\mathscr{D})^{-1} \in \mathcal{K}_{\mathcal{N}}$ for all $\lambda \notin \mathbb{R}$.

The triple is said to be even if there is $\gamma \in \mathcal{N}$ such that $\gamma^{*}=\gamma, \gamma^{2}=1, a \gamma=\gamma a$ for all $a \in \mathcal{A}$ and $\mathscr{D} \gamma+\gamma \mathscr{D}=0$. Otherwise it is odd.

Henceforth we omit the term semifinite as it is implied by the use of a faithful normal semifinite trace on $\mathcal{N}$ in all of the subsequent text. In this paper, for simplicity of exposition, we will deal only with unital algebras $\mathcal{A} \subset \mathcal{N}$ where the identity of $\mathcal{A}$ is that of $\mathcal{N}$.

Definition 2.2. A semifinite spectral triple $(\mathcal{A}, \mathscr{H}, \mathscr{D})$ is $Q C^{k}$ for $k \geq 1(Q$ for quantum) if for all $a \in \mathcal{A}$ the operators $a$ and $[\mathcal{D}, a]$ are in the domain of $\delta^{k}$, where $\delta(T)=[|\mathscr{D}|, T]$ is the partial derivation on $\mathcal{N}$ defined by $|\mathscr{D}|$. We say that $(\mathcal{A}, \mathscr{H}, \mathscr{D})$ is $Q C^{\infty}$ if it is $Q C^{k}$ for all $k \geq 1$.

The notation is meant to be analogous to the classical case, but we introduce the $Q$ so that there is no confusion between quantum differentiability of $a \in \mathcal{A}$ and classical differentiability of functions. We will sometimes use the notation $d a:=[\mathscr{D}, a]$ for the derivation $[\mathscr{D}, \cdot]$.

Partial derivation means that $\delta$ is defined on some subalgebra of $\mathcal{N}$ which need not be (weakly) dense in $\mathcal{N}$. More precisely, $\operatorname{dom} \delta=\{T \in \mathcal{N} \mid \delta(T)$ is bounded $\}$. We also note that if $T \in \mathcal{N}$, one can show that $[|D|, T]$ is bounded if and only if $\left[\left(1+D^{2}\right)^{1 / 2}, T\right]$ is bounded, by using the functional calculus to show that $|\mathscr{D}|-\left(1+\mathscr{D}^{2}\right)^{1 / 2}$ extends to a bounded operator in $\mathcal{N}$. In fact, writing $|\mathscr{D}|_{1}=$ $\left(1+\mathscr{D}^{2}\right)^{1 / 2}$ and $\delta_{1}(T)=\left[|\mathscr{D}|_{1}, T\right]$ we have $\operatorname{dom} \delta^{n}=\operatorname{dom} \delta_{1}^{n}$ for all $n$. Thus the condition defining $Q C^{\infty}$ can be replaced by

$$
a,[D, a] \in \bigcap_{n \geq 0} \operatorname{dom} \delta_{1}^{n} \quad \text { for all } a \in \mathcal{A} .
$$

This is important in situations where we cannot assume $|\mathscr{D}|$ is invertible.

We also observe that if $T \in \mathcal{N}$ and $[\mathscr{D}, T]$ is bounded, then $[\mathscr{D}, T] \in \mathcal{N}$. Similar comments apply to $[|D|, T],\left[\left(1+\mathscr{D}^{2}\right)^{1 / 2}, T\right]$ and the more exotic combinations 
such as $\left[\mathscr{D}^{2}, T\right]\left(1+\mathscr{D}^{2}\right)^{-1 / 2}$ which we will encounter later. The proofs of these statements can be found in [CPRS2].

Recall from [FK] that if $S \in \mathcal{N}$, the $t$-th generalized singular value of $S$ for each real $t>0$ is given by

$$
\mu_{t}(S)=\inf \{\|S E\| \mid E \text { is a projection in } \mathcal{N} \text { with } \tau(1-E) \leq t\} .
$$

The ideal $\mathscr{L}^{1}(\mathcal{N})$ consists of those operators $T \in \mathcal{N}$ such that $\|T\|_{1}:=\tau(|T|)<$ $\infty$ where $|T|=\sqrt{T^{*} T}$. In the Type I setting this is the usual trace class ideal. We will simply write $\mathscr{L}^{1}$ for this ideal in order to simplify the notation and denote the norm on $\mathscr{L}^{1}$ by $\|\cdot\|_{1}$. An alternative definition in terms of singular values is that $T \in \mathscr{L}^{1}$ if $\|T\|_{1}:=\int_{0}^{\infty} \mu_{t}(T) d t<\infty$. Note that in the case where $\mathcal{N} \neq \mathscr{B}(\mathscr{H})$, $\mathscr{L}^{1}$ need not be complete in this norm but it is complete in the norm $\|\cdot\|_{1}+\|\cdot\|_{\infty}$. (where $\|\cdot\|_{\infty}$ is the uniform norm).

2.2. The pseudodifferential calculus. We refer to [CPRS2], Section 6, and [CoM], [Co5] for a full discussion of the pseudodifferential calculus. We summarise here what is needed for this paper. Given a densely-defined self-adjoint unbounded operator $D$ on a Hilbert space $\mathscr{H}$, we set $\mathscr{H}_{\infty}=\bigcap_{n \geq 0}$ dom $D^{n}$. As above we have the partial derivation given by $\delta_{1}(T)=\left[|D|_{1}, T\right]$ with $|D|_{1}=\left(1+D^{2}\right)^{1 / 2}$ and we define linear spaces of operators, for $r \in \mathbb{R}$, by

$$
O P^{0}=\bigcap_{n \geq 0} \operatorname{dom} \delta_{1}^{n}, \quad O P^{r}=\left(1+D^{2}\right)^{r / 2} O P^{0} .
$$

We observe that $\delta_{1}$ leaves $O P^{0}$ invariant. If $(\mathcal{A}, \mathscr{H}, \mathscr{D})$ is a $Q C^{\infty}$ spectral triple and we define $\mathscr{H}_{\infty}$ and the spaces $O P^{r}$ using $\mathscr{D}$, then $\mathcal{A},[D, \mathscr{A}] \subset O P^{0}$. Defining $\nabla(T)=\left[\mathscr{D}^{2}, T\right]$ and setting $T^{(n)}=\nabla^{n}(T)$, we find that $a^{(n)}$ and $[\mathscr{D}, a]^{(n)}$ are in $O P^{n}$ for all $a \in \mathcal{A}$.

We next recall Lemma 6.2 of [CPRS2].

Lemma 2.3 (Compare Lemma 1.1 of [Co5]). Let $b \in O P^{0}, \sigma_{1}(b)=|D|_{1} b|D|_{1}^{-1}$ and $\varepsilon_{1}(b)=\delta_{1}(b)|D|_{1}^{-1}$. Then we have

(1) $\sigma_{1}=\mathrm{Id}+\varepsilon_{1}$,

(2) $\varepsilon_{1}^{n}(b)=\delta_{1}^{n}(b)|D|_{1}^{-n} \in O P^{0}$ for all $n$,

(3) $\sigma_{1}^{n}(b)=\left(\operatorname{Id}+\varepsilon_{1}\right)^{n}(b)=\sum_{k=0}^{n}\left(\begin{array}{l}n \\ k\end{array}\right) \delta_{1}^{k}(b)|D|_{1}^{-k} \in O P^{0}$ for all $n$.

In a similar manner we can also prove the following.

Lemma 2.4. Let $b \in O P^{0}$. With $\gamma_{1}(b)=2 \delta_{1}(b)|D|_{1}$, we have:

(1) $\nabla=\delta_{1}^{2}+\gamma_{1}$,

(2) $\gamma_{1}^{k}(b)=2^{k} \delta_{1}^{k}(b)|D|_{1}^{k} \in O P^{k}$ for all $k$, 
(3) $b^{(n)}:=\nabla^{n}(b)=\left(\sum_{k=0}^{n} 2^{k}\left(\begin{array}{l}n \\ k\end{array}\right) \delta_{1}^{2 n-k}(b)|D|_{1}^{-n+k}\right)|D|_{1}^{n} \in$ OP for all $n$.

Proof. Part (1) is a straightforward calculation noting that $\nabla(b)=\left[|D|_{1}^{2}, b\right]$. Part (2) follows from the definition of $\gamma_{1}$ by induction. Part (3) follows from applying the binomial theorem to (1) and then using (2).

Definition 2.5. We define an increasing sequence of norms $\|\cdot\|_{k}$ on $O P^{0}$ via $\|b\|_{k}=$ $\sum_{j=0}^{k}\left\|\delta_{1}^{j}(b)\right\|$ for $k \geq 0$. This is closely related to the $\delta$-topology on $Q C^{\infty}$ algebras, $\mathcal{A}$, given by the family of seminorms:

$$
a \rightarrow\left\|\delta^{k}(a)\right\|, \quad a \rightarrow\left\|\delta^{k}([\mathcal{D}, a])\right\|, \quad k=0,1,2, \ldots
$$

Remark. For $b \in O P^{0}$, we let $b_{(n)}=\sum_{k=0}^{n} 2^{k}\left(\begin{array}{l}n \\ k\end{array}\right) \delta_{1}^{2 n-k}(b)|D|_{1}^{-n+k}$ so that $b^{(n)}=$ $b_{(n)}|D|_{1}^{n}$ where $b_{(n)} \in O P^{0}$, and $\left\|b_{(n)}\right\| \leq C_{n}\|b\|_{2 n}$ where the constant $C_{n}$ depends only on $n$.

Lemma 2.6. Let $a \in O P^{0}$ and $n, p \geq 0$. Then

(1) $\sigma_{1}^{p}\left(a_{(n)}\right)=\sum_{k=0}^{n} \sum_{j=0}^{p} 2^{k}\left(\begin{array}{l}n \\ k\end{array}\right)\left(\begin{array}{l}p \\ j\end{array}\right) \delta_{1}^{2 n-k+j}(a)|D|_{1}^{-n+k-j}$ is in $O P^{0}$, and

(2) there is a positive constant $C_{p, n}$ depending only on $p$ and $n$ so that $\left\|\sigma_{1}^{p}\left(a_{(n)}\right)\right\| \leq$ $C_{p, n}\|a\|_{2 n+p}$.

Proof. Part (1) is just a calculation combining the previous two lemmas and noting that $\sigma_{1}$ and $\delta_{1}$ not only commute but are both $|D|_{1}^{-1}$-linear. Part (2) follows from part (1) and the fact that $\left\||D|_{1}^{-1}\right\| \leq 1$.

Corollary 2.7. If $b_{0}, b_{1}, \ldots, b_{m}$ are in $O P^{0}$ and $n_{0}, n_{1}, \ldots, n_{m}$ are nonnegative integers with $|n|:=n_{0}+n_{1}+\cdots+n_{m}$, then there is a $C>0$ depending only on $m$ and $|n|$ so that $b_{0}^{\left(n_{0}\right)} b_{1}^{\left(n_{1}\right)} \ldots b_{m}^{\left(n_{m}\right)}=B|D|_{1}^{|n|}$ where $B \in O P^{0}$ and $\|B\| \leq$ $C\left\|b_{0}\right\|_{2|n|}\left\|b_{1}\right\|_{2|n|} \ldots\left\|b_{m}\right\|_{2|n|}$.

Proof. In the notation of the previous remark

$$
\begin{aligned}
b_{0}^{\left(n_{0}\right)} & b_{1}^{\left(n_{1}\right)} \ldots b_{m}^{\left(n_{m}\right)} \\
& =\left(b_{0}\right)_{\left(n_{0}\right)}|D|_{1}^{n_{0}}\left(b_{1}\right)_{\left(n_{1}\right)}|D|_{1}^{n_{1}} \ldots\left(b_{m}\right)_{\left(n_{m}\right)}|D|_{1}^{n_{m}} \\
& =\left(b_{0}\right)_{\left(n_{0}\right)} \sigma_{1}^{n_{0}}\left(\left(b_{1}\right)_{\left(n_{1}\right)}\right) \sigma_{1}^{n_{0}+n_{1}}\left(\left(b_{2}\right)_{\left(n_{2}\right)}\right) \ldots \sigma_{1}^{|n|}\left(\left(b_{m}\right)_{\left(n_{m}\right)}\right)|D|_{1}^{|n|} .
\end{aligned}
$$

The result now follows from the previous lemma with

$$
B=\left(b_{0}\right)_{\left(n_{0}\right)} \sigma_{1}^{n_{0}}\left(\left(b_{1}\right)_{\left(n_{1}\right)}\right) \sigma_{1}^{n_{0}+n_{1}}\left(\left(b_{2}\right)_{\left(n_{2}\right)}\right) \ldots \sigma_{1}^{|n|}\left(\left(b_{m}\right)_{\left(n_{m}\right)}\right) \text {, }
$$

since $\|\cdot\|_{2 k} \leq\|\cdot\|_{2|n|}$ for each $k \leq|n|$. 
A slight variation on the previous corollary is the following.

Corollary 2.8. If $b_{0}, b_{1}, \ldots, b_{m}$ are in $O P^{0}$ and $n_{0}, n_{1}, \ldots, n_{m}$ are nonnegative integers with $|n|:=n_{0}+n_{1}+\cdots+n_{m}$, then there is a $C>0$ depending only on $m$ and $|n|$ so that

$$
b_{0}^{\left(n_{0}\right)} b_{1}^{\left(n_{1}\right)} \ldots b_{k}^{\left(n_{k}\right)} D b_{k+1}^{\left(n_{k+1}\right)} \ldots b_{m}^{\left(n_{m}\right)}=B|D|^{|n|+1}
$$

where $B \in O P^{0}$ and

$$
\|B\| \leq C\left\|b_{0}\right\|_{2|n|+1}\left\|b_{1}\right\|_{2|n|+1} \ldots\left\|b_{m}\right\|_{2|n|+1} .
$$

Proof. Write $D=F_{0}|D|_{1}$ where $F_{0}:=D|D|_{1}^{-1} \in O P^{0}$ and proceed as in the previous proof.

If $\mathscr{D}$ is $n$-summable, so $(\lambda-\mathscr{D})^{-1} \in \mathscr{L}^{n}(\mathcal{N})$ for all $\lambda \notin \mathbb{R}$, then any $T \in O P^{r}$ is $n / r$-summable.

\section{Cyclic cohomology and Chern characters}

A major feature of [Co4] is the association to a suitable representative of a $K$-theory class, respectively a $K$-homology class, a class in periodic cyclic homology, respectively a class in periodic cyclic cohomology, called a Chern character in both cases. The principal result is then

$$
\langle[x],[(\mathcal{A}, \mathscr{H}, \mathscr{D})]\rangle=\left\langle\left[\mathrm{Ch}_{*}(x)\right],\left[\mathrm{Ch}^{*}(\mathcal{A}, \mathscr{H}, \mathscr{D})\right]\right\rangle,
$$

where $[x] \in K_{*}(\mathcal{A})$ is a $K$-theory class with representative $x$ and $[(\mathcal{A}, \mathscr{H}, \mathscr{D})]$ is the $K$-homology class of the spectral triple $(\mathcal{A}, \mathscr{H}, \mathscr{D})$. (The exact normalisations for these pairings depends on what kind of cochains one uses to represent cyclic cohomology.) On the right-hand side, $\mathrm{Ch}_{*}(x)$ is the Chern character of $x$ and $\left[\mathrm{Ch}_{*}(x)\right]$ its periodic cyclic homology class. Similarly $\left[\mathrm{Ch}^{*}(\mathcal{A}, \mathcal{H}, \mathfrak{D})\right]$ is the periodic cyclic cohomology class of the Chern character of $(\mathscr{A}, \mathscr{H}, \mathscr{D})$. We will describe below the complexes defining cyclic cohomology (the cyclic complex and the $(b, B)$ bicomplex) and the Chern character.

To define the (normalised) $(b, B)$ bicomplex, we introduce the following linear spaces, [Lo]. Let $C_{m}=\mathcal{A} \otimes \overline{\mathcal{A}}^{\otimes m}$ where $\overline{\mathcal{A}}$ is the quotient $\mathcal{A} / \mathbb{C} \cdot I$ with $I$ being the identity element of $\mathcal{A}$ and, assuming with no loss of generality that $\mathcal{A}$ is complete in the $\delta$-topology, [R], we employ the projective tensor product. Let $C^{m}=\operatorname{Hom}\left(C_{m}, \mathbb{C}\right)$ be the linear space of continuous linear functionals on $C_{m}$. We may define the $(b, B)$ bicomplex using these spaces (as opposed to $C_{m}=\mathcal{A}^{\otimes m+1}$ et cetera) and the resulting cohomology will be the same. This follows because the bicomplex defined using 
$\mathcal{A} \otimes \overline{\mathcal{A}}^{\otimes m}$ is quasi-isomorphic to that defined using $\mathscr{A} \otimes \mathcal{A}^{\otimes m}$ [Lo]. Similar comments apply to the cyclic complex.

We first define cyclic cohomology using the cyclic complex. A normalised cyclic cochain on $\mathscr{A}$ is a functional $\psi \in C^{m}$ such that

$$
\psi\left(a_{0}, \ldots, a_{m}\right)=(-1)^{m} \psi\left(a_{m}, a_{0}, \ldots, a_{m-1}\right) .
$$

The set of all normalised cyclic cochains in $C^{m}$ is denote $C_{\lambda}^{m}$. We say that $\psi$ is a cyclic cocycle if for all $a_{0}, \ldots, a_{m+1} \in \mathcal{A}$ we have $(b \psi)\left(a_{0}, \ldots, a_{m+1}\right)=0$ where

$$
\begin{aligned}
& (b \psi)\left(a_{0}, a_{1}, \ldots, a_{m+1}\right) \\
& =\sum_{j=0}^{m}(-1)^{j} \psi\left(a_{0}, a_{1}, \ldots, a_{j} a_{j+1}, \ldots, a_{m+1}\right)+(-1)^{m+1} \phi\left(a_{m+1} a_{0}, a_{1}, \ldots, a_{m}\right) .
\end{aligned}
$$

Then $H_{\lambda}^{m}(\mathcal{A})$, the $m$-th cohomology group of $\left(C_{\lambda}^{m}, b\right)$, is defined to be the $m$-th cyclic cohomology group of $\mathcal{A}$.

The cup product with the generator $\sigma \in H_{\lambda}^{2}(\mathbb{C})$ of the cyclic cohomology of $\mathbb{C}$ defines a map $S: H_{\lambda}^{m}(\mathcal{A}) \rightarrow H_{\lambda}^{m+2}(\mathcal{A})$ for any (locally convex) algebra $\mathcal{A}$. This periodicity operator allows us to define the periodic cyclic cohomology of $\mathcal{A}$ as the direct limit of the cyclic cohomology groups,

$$
H_{\mathrm{per}}^{*}(\mathcal{A})=\lim _{\rightarrow}\left(H_{\lambda}^{m}(\mathcal{A}), S\right),
$$

where $*$ on the left-hand side takes only the values even or odd, and on the right-hand side we consider only those $m$ with the same parity as $*$.

A normalised $(b, B)$-cochain $\phi$ is a finite collection of multilinear functionals,

$$
\phi=\left\{\phi_{m}\right\}_{m=0,1, \ldots, M} \text { with } \phi_{m} \in C^{m} .
$$

An odd cochain has $\phi_{m}=0$ for even $m$, while an even cochain has $\phi_{m}=0$ for odd $m$. It is a (normalised) ( $b, B)$-cocycle if, for all $m, b \phi_{m}+B \phi_{m+2}=0$ where $b: C^{m} \rightarrow C^{m+1}, B: C^{m} \rightarrow C^{m-1}$ are the coboundary operators. We defined $b$ above while $B$ is given by

$$
\begin{aligned}
& \left(B \phi_{m}\right)\left(a_{0}, a_{1}, \ldots, a_{m-1}\right) \\
& \quad=\sum_{j=0}^{m-1}(-1)^{(m-1) j} \phi_{m}\left(1, a_{j}, a_{j+1}, \ldots, a_{m-1}, a_{0}, \ldots, a_{j-1}\right)
\end{aligned}
$$

We write $(b+B) \phi=0$ for brevity. Thought of as functionals on $\mathcal{A}^{\otimes m+1}$ a normalised cocycle will satisfy $\phi\left(a_{0}, a_{1}, \ldots, a_{n}\right)=0$ whenever any $a_{j}=1$ for $j \geq 1$.

Similarly, a $\left(b^{T}, B^{T}\right)$-chain $c$ is a (possibly infinite) collection $c=\left\{c_{m}\right\}_{m=0,1, \ldots}$ with $c_{m} \in C_{m}$. The $(b, B)$-chain $\left\{c_{m}\right\}$ is a $\left(b^{T}, B^{T}\right)$-cycle if $b^{T} c_{m+2}+B^{T} c_{m}=0$ 
for all $m$. More briefly, we write $\left(b^{T}+B^{T}\right) c=0$. Here $b^{T}, B^{T}$ are the boundary operators of cyclic homology and are the transpose of the coboundary operators $b, B$ in the following sense.

The pairing between a $(b, B)$-cochain $\phi=\left\{\phi_{m}\right\}_{m=0}^{M}$ and a $\left(b^{T}, B^{T}\right)$-chain $c=$ $\left\{c_{m}\right\}$ is given by

$$
\langle\phi, c\rangle=\sum_{m=0}^{M} \phi_{m}\left(c_{m}\right) .
$$

This pairing satisfies $\langle(b+B) \phi, c\rangle=\left\langle\phi,\left(b^{T}+B^{T}\right) c\right\rangle$. All of the cocycles we consider in this paper are in fact defined as functionals on $\bigoplus_{m} \mathcal{A} \otimes \overline{\mathcal{A}}^{\otimes m}$. Henceforth we will drop the superscript on $b^{T}, B^{T}$ and just write $b, B$ for both boundary and coboundary operators as the meaning will be clear from the context.

Next we introduce finitely summable Fredholm modules and their Chern characters.

Definition 3.1. A pre-Fredholm module for a unital $*$-algebra $\mathcal{A}$ is a pair $(\mathscr{H}, F)$ where $\mathcal{A}$ is represented in $\mathcal{N}$ (a semifinite von Neumann algebra acting on $\mathscr{H}$ with fixed trace $\tau$ ) and $F$ is a self-adjoint Breuer-Fredholm operator in $\mathcal{N}$ satisfying:

1. $1-F^{2} \in \mathcal{K}_{\mathcal{N}}$, and

2. $[F, a] \in \mathcal{K}_{\mathcal{N}}$ for $a \in \mathcal{A}$.

We say that $(\mathcal{H}, F)$ is even if there is a grading operator $\gamma \in \mathcal{N}$ such that $\gamma^{*}=\gamma$, $\gamma^{2}=1,[\gamma, a]=0$ for all $a \in \mathcal{A}$ and $\gamma F+F \gamma=0$; otherwise, $(\mathcal{H}, F)$ is odd. Our formulas will often include a factor of $\gamma$ in the odd case as well as the even case: in the odd case we interpret $\gamma$ to be 1 . If $1-F^{2}=0$ we drop the prefix "pre-". If $[F, a] \in \mathscr{L}^{p}(\mathcal{N})$ for all $a \in \mathcal{A}$, we say that $(\mathcal{H}, F)$ is $p$-summable.

Pertinent example. Semifinite spectral triples give rise to pre-Fredholm modules via

$$
(\mathscr{A}, \mathscr{H}, \mathscr{D}) \mapsto\left(\mathscr{H}, F=\mathscr{D}\left(1+\mathscr{D}^{2}\right)^{-1 / 2}\right) .
$$

One views spectral triples as geometric representatives of $K$-homology classes in much the same way that one views differential forms as geometric representatives of cohomology classes.

If the semifinite spectral triple $(\mathscr{A}, \mathscr{H}, \mathscr{D})$ is $Q C^{\infty}$ and finitely summable with

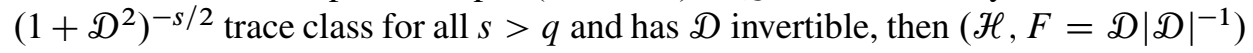
is a $[q]+1$-summable Fredholm module where $[\cdot]$ denotes the integer part.

Definition 3.2. We define the 'conditional trace' $\tau^{\prime}$ by

$$
\tau^{\prime}(T)=\frac{1}{2} \tau(F(F T+T F)),
$$


provided that $F T+T F \in \mathscr{L}^{1}(\mathcal{N})$ (as it will be in our case; see [Co4], p. 293). Note that if $T \in \mathscr{L}^{1}(\mathcal{N})$ we have (using the trace property and $F^{2}=1$ )

$$
\tau^{\prime}(T)=\tau(T) .
$$

The Chern character $\left[\mathrm{Ch}_{F}\right]$ of an $n+1$-summable Fredholm module $(\mathscr{H}, F)$ ( $n$ an integer) is the class in periodic cyclic cohomology of the cyclic cocycles

$$
\lambda_{m} \tau^{\prime}\left(\gamma a_{0}\left[F, a_{1}\right] \ldots\left[F, a_{m}\right]\right),
$$

with $a_{0}, \ldots, a_{m} \in \mathcal{A}, m \geq n, m$ even if $(\mathcal{H}, F)$ even, and odd otherwise. Here $\lambda_{m}$ are constants ensuring that this collection of cocycles yields a well-defined periodic class, and they are given by

$$
\lambda_{m}= \begin{cases}(-1)^{m(m-1) / 2} \Gamma\left(\frac{m}{2}+1\right) & m \text { even, } \\ \sqrt{2 i}(-1)^{m(m-1) / 2} \Gamma\left(\frac{m}{2}+1\right) & m \text { odd. }\end{cases}
$$

The class of the Chern character of an $n+1$-summable Fredholm module is represented by the cyclic cocycle in bottom dimension $n, \mathrm{Ch}_{F} \in C_{\lambda}^{n}(\mathcal{A})$ :

$$
\mathrm{Ch}_{F}\left(a_{0}, \ldots, a_{n}\right)=\lambda_{n} \tau^{\prime}\left(\gamma a_{0}\left[F, a_{1}\right] \ldots\left[F, a_{n}\right]\right), \quad a_{0}, \ldots, a_{n} \in \mathcal{A} .
$$

We will always take the cyclic cochain $\mathrm{Ch}_{F}$ (or its $(b, B)$ analogue; see below) as representative of $\left[\mathrm{Ch}_{F}\right]$ and will often refer to $\mathrm{Ch}_{F}$ as the Chern character.

Since the Chern character is a cyclic cochain, it lies in the image of the operator $B$, [Co4], Corollary 20, III.1. $\beta$, and so $B \mathrm{Ch}_{F}=0$ since $B^{2}=0$. Since $b \mathrm{Ch}_{F}=0$, we may regard the Chern character as a one term element of the $(b, B)$ bicomplex. However, the correct normalisation is (taking the Chern character to be in degree $n$ )

$$
C_{\lambda}^{n} \ni \mathrm{Ch}_{F} \mapsto \frac{(-1)^{[n / 2]}}{n !} \mathrm{Ch}_{F} \in C^{n} .
$$

Thus instead of $\lambda_{n}$ defined above, we use $\mu_{n}$

$$
\mu_{n}=\frac{(-1)^{[n / 2]}}{n !} \lambda_{n}= \begin{cases}\frac{\Gamma\left(\frac{n}{2}+1\right)}{n !} & n \text { even, } \\ \sqrt{2 i \frac{\Gamma\left(\frac{n}{2}+1\right)}{n !}} & n \text { odd. }\end{cases}
$$

The difference in normalisation between periodic and $(b, B)$ is due to the way the index pairing is defined in the two cases, [Co4], and compatibility with the periodicity operator.

Our next task is to show that if our spectral triple $(\mathcal{A}, \mathscr{H}, \mathscr{D})$ is such that $\mathscr{D}$ is not invertible, we can replace it by a new spectral triple in the same $K$-homology class in which the unbounded operator is invertible. This is not a precise statement in the 
general semifinite case, as our spectral triples will not define $K$-homology classes in the usual sense. When we say that two spectral triples are in the same $K$-homology class, we shall take this to mean that the associated pre-Fredholm modules are operator homotopic up to the addition of degenerate Fredholm modules (see $[\mathrm{K}]$ for these notions, which make sense in our context).

Definition 3.3. Let $(\mathcal{A}, \mathscr{H}, \mathscr{D})$ be a spectral triple. For any $\mu \in \mathbb{R} \backslash\{0\}$, define the 'double' of $(\mathscr{A}, \mathscr{H}, \mathscr{D})$ to be the spectral triple $\left(\mathcal{A}, \mathscr{H}^{2}, \mathscr{D}_{\mu}\right)$ with $\mathscr{H}^{2}=\mathscr{H} \oplus \mathscr{H}$, and the action of $\mathscr{A}$ and $\mathscr{D}_{\mu}$ given by

$$
\mathscr{D}_{\mu}=\left(\begin{array}{cc}
\mathscr{D} & \mu \\
\mu & -\mathscr{D}
\end{array}\right), \quad a \mapsto\left(\begin{array}{cc}
a & 0 \\
0 & 0
\end{array}\right) \quad \text { for all } a \in \mathcal{A}
$$

Remark. Whether $\mathscr{D}$ is invertible or not, $\mathscr{D}_{\mu}$ always is invertible and $F_{\mu}=\mathscr{D}_{\mu}\left|\mathscr{D}_{\mu}\right|^{-1}$ has square 1. This is the chief reason for introducing this construction.

Lemma 3.4. The $K$-homology classes of $(\mathcal{A}, \mathscr{H}, \mathscr{D})$ and $\left(\mathcal{A}, \mathscr{H}^{2}, \mathscr{D}_{\mu}\right)$ are the same. A representative of this class is $\left(\mathscr{H}^{2}, F_{\mu}\right)$ with $F_{\mu}=\mathscr{D}_{\mu}\left|\mathscr{D}_{\mu}\right|^{-1}$.

The most basic consequence of Lemma 3.4, whose proof may be found in [CPRS1], comes from the following (see [Co1], pp. 65-68, for the proof).

Proposition 3.5. The periodic cyclic cohomology class of the Chern character of a finitely summable Fredholm module depends only on its K-homology class.

In particular, therefore the Chern characters of $(\mathcal{A}, \mathscr{H}, \mathscr{D})$ and $\left(\mathcal{A}, \mathscr{H}^{2}, D_{\mu}\right)$ have the same class in periodic cyclic cohomology, and this can be computed (indeed is defined!) using the Fredholm module $\left(\mathcal{H}^{2}, F_{\mu}\right)$, and this class is independent of $\mu$.

\section{The theorem on the residue cocycle}

4.1. The odd semifinite local index formula. Our main results in this paper are motivated by, and have consequences for, the semifinite local index formula in the odd case [CPRS2]. To state it we need some notation, beginning with multi-indices $\left(k_{1}, \ldots, k_{m}\right), k_{i}=0,1,2, \ldots$, whose length $m$ will always be clear from the context. Next we write $|k|=k_{1}+\cdots+k_{m}$ and define $\alpha(k)$ by

$$
\alpha(k)=\left(k_{1} ! k_{2} ! \ldots k_{m} !\left(k_{1}+1\right)\left(k_{1}+k_{2}+2\right) \ldots(|k|+m)\right)^{-1} .
$$

The numbers $\sigma_{n, j}$ are defined by the equality

$$
\prod_{j=0}^{n-1}(z+j+1 / 2)=\sum_{j=0}^{n} z^{j} \sigma_{n, j} .
$$


If $(\mathcal{A}, \mathscr{H}, \mathscr{D})$ is a $Q C^{\infty}$ spectral triple and $T \in \mathcal{N}$, we write $T^{(n)}$ to denote the iterated commutator $\left[\mathscr{D}^{2},\left[\mathscr{D}^{2},\left[\ldots,\left[\mathscr{D}^{2}, T\right] \ldots\right]\right]\right]$ where we have $n$ commutators with $\mathscr{D}^{2}$. It follows from the remarks after Definition 2.2 that operators of the form

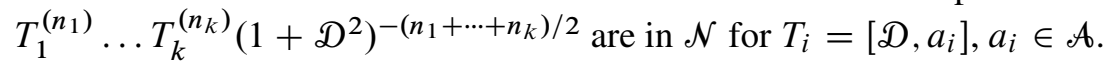

Definition 4.1. If $(\mathcal{A}, \mathcal{H}, \mathscr{D})$ is a $Q C^{\infty}$ spectral triple, we call

$$
q=\inf \left\{k \in \mathbb{R} \mid \tau\left(\left(1+D^{2}\right)^{-k / 2}\right)<\infty\right\}
$$

the spectral dimension of $(\mathcal{A}, \mathscr{H}, \mathscr{D})$. We say that $(\mathscr{A}, \mathscr{H}, \mathscr{D})$ has isolated spectral dimension if for $b$ of the form

$$
b=a_{0}\left[\mathscr{D}, a_{1}\right]^{\left(k_{1}\right)} \ldots\left[\mathscr{D}, a_{m}\right]^{\left(k_{m}\right)}\left(1+\mathscr{D}^{2}\right)^{-m / 2-|k|}
$$

the zeta functions

$$
\zeta_{b}(z-(1-q) / 2)=\tau\left(b\left(1+\mathscr{D}^{2}\right)^{-z+(1-q) / 2}\right)
$$

have analytic continuations to a deleted neighbourhood of $z=(1-q) / 2$.

Remark. We allow the possibility that the analytic continuations of these zeta functions may have an essential singularity at $z=(1-q) / 2$ requiring only that the residues at this point exist. Note that discrete dimension spectrum implies isolated spectral dimension.

Definition 4.2. Now we define, for $(\mathcal{A}, \mathcal{H}, \mathscr{D})$ having isolated spectral dimension and

$$
b=a_{0}\left[\mathscr{D}, a_{1}\right]^{\left(k_{1}\right)} \ldots\left[\mathscr{D}, a_{m}\right]^{\left(k_{m}\right)}\left(1+\mathscr{D}^{2}\right)^{-m / 2-|k|},
$$

the numbers $\tau_{j}(b)$ by:

$$
\tau_{j}(b)=\operatorname{res}_{z=(1-q) / 2}(z-(1-q) / 2)^{j} \zeta_{b}(z-(1-q) / 2) .
$$

Let $Q$ be the spectral projection of $\mathscr{D}$ corresponding to the interval $[0, \infty)$. In [CPRS2] we proved the following result:

Theorem 4.3 (Odd semifinite local index formula). Let $(\mathcal{A}, \mathscr{H}, \mathcal{D})$ be an odd finitely summable $Q C^{\infty}$ spectral triple with spectral dimension $q \geq 1$. Let $N=[q / 2]+1$ where $[\cdot]$ denotes the integer part, and let $u \in \mathcal{A}$ be unitary. Then

(1) $\operatorname{index}(Q u Q)=\frac{1}{\sqrt{2 \pi i}} \operatorname{res}_{r=(1-q) / 2}\left(\sum_{m=1, \mathrm{odd}}^{2 N-1} \phi_{m}^{r}\left(\mathrm{Ch}_{m}(u)\right)\right)$ where for $a_{0}$, $\ldots, a_{m} \in \mathcal{A}, l=\{a+i v \mid v \in \mathbb{R}\}, 0<a<1 / 2, R_{s}(\lambda)=\left(\lambda-\left(1+s^{2}+\mathscr{D}^{2}\right)\right)^{-1}$ and $r>0$ we define $\phi_{m}^{r}\left(a_{0}, a_{1}, \ldots, a_{m}\right)$ to be

$$
\begin{aligned}
& \frac{-2 \sqrt{2 \pi i}}{\Gamma((m+1) / 2)} \int_{0}^{\infty} s^{m} \tau\left(\frac{1}{2 \pi i} \int_{l} \lambda^{-q / 2-r} a_{0} R_{s}(\lambda)\left[\mathcal{D}, a_{1}\right] R_{s}(\lambda)\right. \\
& \left.\ldots\left[\mathscr{D}, a_{m}\right] R_{s}(\lambda) d \lambda\right) d s .
\end{aligned}
$$


In particular the sum on the right-hand side of (1) analytically continues to a deleted neighbourhood of $r=(1-q) / 2$ with at worst a simple pole at $r=(1-q) / 2$. Moreover, the complex function-valued cochain $\left(\phi_{m}^{r}\right)_{m=1 \text {,odd }}^{2 N-1}$ is $a(b, B)$ cocycle for $A$ modulo functions holomorphic in a half-plane containing $r=(1-q) / 2$.

(2) The index is also the residue of a sum of zeta functions:

$$
\begin{aligned}
& \frac{1}{\sqrt{2 \pi i}} \operatorname{res}_{r=(1-q) / 2}\left(\sum_{m=1, \mathrm{odd}}^{2 N-1} \sum_{|k|=0}^{2 N-1-m} \sum_{j=0}^{|k|+(m-1) / 2}(-1)^{|k|+m} \alpha(k) \Gamma((m+1) / 2)\right. \\
& \text { - } \sigma_{|k|+(m-1) / 2, j}(r-(1-q) / 2)^{j} \tau\left(u^{*}[\mathscr{D}, u]^{\left(k_{1}\right)}\left[\mathcal{D}, u^{*}\right]^{\left(k_{2}\right)}\right. \\
& \left.\left.\ldots[\mathscr{D}, u]^{\left(k_{m}\right)}\left(1+\mathscr{D}^{2}\right)^{-m / 2-|k|-r+(1-q) / 2}\right)\right) .
\end{aligned}
$$

In particular the sum of zeta functions on the right-hand side analytically continues to a deleted neighbourhood of $r=(1-q) / 2$ and has at worst a simple pole at $r=(1-q) / 2$.

(3) If $(\mathcal{A}, \mathscr{H}, \mathscr{D})$ also has isolated spectral dimension then

$$
\operatorname{index}(Q u Q)=\frac{1}{\sqrt{2 \pi i}} \sum_{m} \phi_{m}\left(\mathrm{Ch}_{m}(u)\right)
$$

where for $a_{0}, \ldots, a_{m} \in \mathcal{A}$

$$
\begin{aligned}
& \phi_{m}\left(a_{0}, \ldots, a_{m}\right)=\operatorname{res}_{r=(1-q) / 2} \phi_{m}^{r}\left(a_{0}, \ldots, a_{m}\right) \\
& =\sqrt{2 \pi i} \sum_{|k|=0}^{2 N-1-m}(-1)^{|k|} \alpha(k) \sum_{j=0}^{|k|+(m-1) / 2} \sigma_{(|k|+(m-1) / 2), j} \tau_{j}\left(a_{0}\left[\mathscr{D}, a_{1}\right]^{\left(k_{1}\right)}\right. \\
& \left.\ldots\left[\mathscr{D}, a_{m}\right]^{\left(k_{m}\right)}\left(1+\mathscr{D}^{2}\right)^{-|k|-m / 2}\right),
\end{aligned}
$$

and $\left(\phi_{m}\right)_{m=1, \text { odd }}^{2 N-1}$ is a $(b, B)$ cocycle for $\mathcal{A}$. When $q<2 N-1$, the term with $m=2 N-1$ is zero, and for $m=1,3, \ldots, 2 N-3$, all the top terms with $|k|=2 N-1-m$ are zero.

In [CPRS3] we established the even version of this theorem however we will have no need to state the full result here.

4.2. The resolvent cocycle in the general case. We extract from the preceding theorem the following definition of the resolvent cocycle. It treats the even and odd cases in the same fashion. It should be compared with the JLO cocycle (the foundation of the original Connes-Moscovici local index theorem). The more general version of the local index formula that we obtained exploits the algebraic and analytic properties 
of this resolvent cocycle. We will describe these carefully. (Note that we derived this cocycle differently in the odd and even cases, from the spectral flow formula of [CP2] in the odd case and from a generalised McKean-Singer formula in the even case [CPRS3].)

Definition 4.4. Let $(\mathcal{A}, \mathscr{H}, \mathscr{D})$ be a spectral triple with spectral dimension $q \geq 1$. Let $P$ denote the parity of the triple, so $P=0$ for even triples and $P=1$ for odd triples. Let $A$ denote $(P-1)$, the anti-parity, so $A=1$ for even triples and $A=0$ for odd triples. We adopt the convention that $|\mathscr{D}|$ and elements of $\mathcal{A}$ have grading degree zero, while $\mathscr{D}$ has grading degree one. In the even case this is of course the actual grading degree of the spectral triple. We denote the grading degree of $T \in O P^{*}$ by $\operatorname{deg}(T)$. Finally, let $N=[(q+1+P) / 2]$ where [ $]$ denotes the integer part. So $M=2 N-P$ is the greatest integer of parity $P$ in $q+1$. In particular, if $q$ is an integer of parity $P$ then $M=2 N-P=q$.

The grading degree is used to define the graded commutator:

$$
[T, R]_{ \pm}:=T R-(-1)^{\operatorname{deg}(T) \operatorname{deg}(R)} R T .
$$

In particular, we have $[\mathscr{D}, a]_{ \pm}=[\mathscr{D}, a]$ for all $a \in \mathcal{A}$. Similarly, since $\operatorname{deg}\left(\mathscr{D}^{2}\right)=0$, we have $\left[\mathscr{D}^{2}, T\right]_{ \pm}=\left[\mathscr{D}^{2}, T\right]$ for all $T$. The following definition generalises the expectations introduced in [CPRS2], [CPRS3] to deal with both the even and odd cases in a uniform fashion.

Definition 4.5. Let $0<a<1 / 2$ and let $l$ be the vertical line $l=\{a+i v \mid v \in \mathbb{R}\}$. For $m \geq 0, s \in[0, \infty)$ and operators $A_{0}, \ldots, A_{m}, A_{i} \in O P^{k_{i}}$, with $k_{0}+\cdots+k_{m}-2 m<$ $2 \operatorname{Re}(r)$ define

$$
\left\langle A_{0}, \ldots, A_{m}\right\rangle_{m, s, r}=\tau\left(\frac{1}{2 \pi i} \gamma \int_{l} \lambda^{-q / 2-r} A_{0} R_{s}(\lambda) A_{1} \ldots A_{m} R_{S}(\lambda) d \lambda\right) .
$$

Here $\gamma$ is the $\mathbb{Z}_{2}$-grading in the even case and the identity operator in the odd case, and $R_{S}(\lambda)=\left(\lambda-\left(1+s^{2}+\mathscr{D}^{2}\right)\right)^{-1}$.

We now state the definition of the resolvent cocycle in terms of the expectations $\langle\ldots\rangle_{m, s, r}$.

Definition 4.6. (Compare Definition 7.9 [CPRS2]) Let $(\mathcal{A}, \mathscr{H}, \mathscr{D})$ be a spectral triple with spectral dimension $q \geq 1$ and parity $P$. Introduce constants $\eta_{m}, m=0,1,2, \ldots$ with $P \equiv m(\bmod 2)$ by

$$
\eta_{m}=(-\sqrt{2 i})^{P} 2^{m+1} \frac{\Gamma(m / 2+1)}{\Gamma(m+1)} .
$$


Then for $\operatorname{Re}(r)>\frac{1}{2}(1-m)$ the $m$-th component of the resolvent cocycle $\phi_{m}^{r}: \mathcal{A}^{\otimes m+1} \rightarrow \mathbb{C}$ is defined by:

$$
\phi_{m}^{r}\left(a_{0}, \ldots, a_{m}\right)=\eta_{m} \int_{0}^{\infty} s^{m}\left\langle a_{0}, d a_{1}, \ldots, d a_{m}\right\rangle_{m, s, r} d s
$$

where recall that $d a:=[\mathscr{D}, a]$ for $a \in \mathcal{A}$.

A basic result which is implicit in [CPRS2], [CPRS3] is that $\phi_{m}^{r}$ is a continuous map from $\mathcal{A}^{\otimes m+1}$ with the $\delta$-topology (see Definition 2.5) to the space of functions $F_{m}$ defined and holomorphic in the half-plane $\operatorname{Re}(r)>(1-m) / 2$ (with the topology of uniform convergence on compacta). This turns out to be surprisingly subtle, so we include the proof with a slight generalisation which we will require a little later. Let $R_{s, t}(\lambda):=\left(\lambda-\left(t+s^{2}+\mathscr{D}^{2}\right)\right)^{-1}$ where if $\mathscr{D}^{2} \geq \delta>0$ (and so invertible) we allow $t \in[0,1]$ and if $\mathscr{D}$ is not invertible we allow only $t=1$. Define $\phi_{m, t}^{r}$ just as $\phi_{m}^{r}$, but using $R_{s, t}(\lambda)$ in place of $R_{s}(\lambda)$, and similarly for $\langle\ldots\rangle_{m, s, r, t}$.

Lemma 4.7. Let $O P^{0}$ have the $\delta$-topology (see Definition 2.5). Then with $t \in[0,1]$ as above, the map

$$
\left(O P^{0}\right)^{\otimes m+1} \ni\left(A_{0}, \ldots, A_{m}\right) \mapsto \int_{0}^{\infty} s^{m}\left\langle A_{0}, \ldots, A_{m}\right\rangle_{m, s, r, t} d s \in F_{m}
$$

is a continuous multilinear functional. In particular for each $r \in \mathbb{C}$ with $\operatorname{Re}(r)>$ $(1-m) / 2, \phi_{m, t}^{r}$ restricts to a continuous multilinear functional on $\mathcal{A}$ with the $\delta$-topology. Moreover, $\left(a_{0}, a_{1}, \ldots, a_{m}\right) \mapsto\left(r \mapsto \phi_{m, t}^{r}\left(a_{0}, \ldots, a_{m}\right)\right)$ is an element of $\operatorname{Hom}\left(\mathcal{A}^{\otimes m+1}, F_{m}\right)$.

Proof. Since $\mathcal{A},[\mathscr{D}, \mathcal{A}] \subset O P^{0}$, the penultimate statement of the lemma follows easily from the first. To prove the first statement we begin by rewriting the expectations $\langle\ldots\rangle_{m, s, r, t}$ using both the $s$-trick (see Lemma 5.6 below) and the $\lambda$-trick (see Lemma 5.7 below). The $s$-trick says that for all $A_{0}, \ldots, A_{m} \in O P^{0}$ we have

$$
\begin{aligned}
\int_{0}^{\infty} s^{m}\left\langle A_{0}, \ldots, A_{m}\right\rangle_{m, s, r, t} d s \\
\quad=\frac{-2}{m+1} \sum_{j=0}^{m} \int_{0}^{\infty} s^{m+2}\left\langle A_{0}, \ldots, A_{j}, 1, A_{j+1}, \ldots, A_{m}\right\rangle_{m+1, s, r, t},
\end{aligned}
$$

where both sides exist for $\operatorname{Re}(r)>(1-m) / 2$. On the other hand, the $\lambda$-trick says that for all $A_{0}, \ldots, A_{m} \in O P^{0}$ we have

$$
\begin{aligned}
\int_{0}^{\infty} s^{m} & \left\langle A_{0}, \ldots, A_{m}\right\rangle_{m, s, r, t} d s \\
& =\frac{-1}{q / 2+r-1} \sum_{j=0}^{m} \int_{0}^{\infty} s^{m}\left\langle A_{0}, \ldots, A_{j}, 1, A_{j+1}, \ldots, A_{m}\right\rangle_{m+1, s, r-1, t},
\end{aligned}
$$


where both sides exist for $\operatorname{Re}(r)>(1-m) / 2$ provided the first factor on the right does not introduce a pole in this region.

\section{Some simple observations}

(1) The $s$-trick and the $\lambda$-trick commute.

(2) Both tricks leave the region of convergence unaffected, with the proviso that we don't introduce a pole with the $\lambda$-trick.

(3) After $X$ combined applications of the two tricks, the term with any one particular pattern of $1^{\prime} s$ dispersed among the $A_{i}$ 's will appear with frequency exactly $X$ !.

Let $M=2 N-P$ so that $m$ also has parity $P$. If $M=m$ we do nothing to the expression for $\phi_{m, t}^{r}$. Assuming $m<M$ we will apply the $s$-trick exactly $(M-m) / 2$ times (which will raise the power of $s^{m}$ to $s^{M}$ and no more) and then the $\lambda$-trick also exactly $(M-m) / 2$ times, which will raise our $m+1$-tuple to an $M+1$-tuple without introducing a pole in the region $\operatorname{Re}(r)>(1-m) / 2$.

Thus, we obtain by summing over all $k=\left(k_{0}, \ldots, k_{m}\right)$ where each $0 \leq k_{i} \leq$ $(M-m)$ and $|k|=(M-m)$ :

$$
\begin{aligned}
& \int_{0}^{\infty} s^{m}\left\langle A_{0}, \ldots, A_{m}\right\rangle_{m, s, r, t} d s \\
&=(2)^{\frac{M-m}{2}}(M-m) ! \prod_{b=1}^{\frac{M-m}{2}} \frac{1}{q / 2+r-b} \prod_{j=1}^{\frac{M-m}{2}} \frac{1}{m+j} \\
& \quad \cdot \sum_{k} \int_{0}^{\infty} s^{M}\left\langle A_{0}, 1^{k_{0}}, A_{1}, 1^{k_{1}}, \ldots, A_{m}, 1^{k_{m}}\right\rangle_{M, s, r-(M-m) / 2, t} d s
\end{aligned}
$$

where, of course, we mean $1^{k_{i}}=1,1, \ldots, 1$ with $k_{i}$ one's. We observe that both sides converge for $\operatorname{Re}(r)>(1-m) / 2$. Now the poles occur when $r=b-q / 2$ and since $b \leq(M-m) / 2$ and $M \leq q+1$ we have for such poles, $r \leq(M-m) / 2-$ $q / 2 \leq(q+1-m) / 2-q / 2=(1-m) / 2$ so that the pole is not in the region, $\operatorname{Re}(r)>(1-m) / 2$. That is, both sides exist in this region.

Now ignoring the prefactors, we have a sum of integrals where we write $R$ for $R_{s, t}(\lambda)$ and each $n_{i}=k_{i}+1$ so that $n=\left(n_{0}, \ldots, n_{m}\right)$ with $1 \leq n_{i} \leq(M-m)+1$ and $|n|=M+1$ :

$$
\sum_{n} \int_{0}^{\infty} s^{M} \tau\left(\frac{1}{2 \pi i} \gamma \int_{l} \lambda^{-q / 2-r+(M-m) / 2} A_{0} R^{n_{0}} A_{1} R^{n_{1}} \ldots A_{m} R^{n_{m}} d \lambda\right) d s
$$

By Lemma 2.2 of [CP1] we can estimate the trace of the $\lambda$-integral by integrating the trace norm of the integrand. 
For $n$ fixed let $p_{n_{i}}=(M+1) / n_{i}$ so that $\sum_{i} p_{n_{i}}=1$ and using Hölder's inequality we estimate:

$$
\begin{aligned}
\sum_{n} \| A_{0} & R^{n_{1}} A_{1} R^{n_{2}} \ldots R^{n_{m}} A_{m} R^{n_{m+1}} \|_{1} \\
& \leq \sum_{n}\left\|A_{0} R^{n_{1}}\right\|_{p_{n_{i}}} \ldots\left\|A_{m} R^{n_{m+1}}\right\|_{p_{n_{m+1}}} \\
& \leq \sum_{n}\left\|A_{0}\right\| \ldots\left\|A_{m}\right\| \cdot\left\|R^{n_{1}}\right\|_{p_{n_{1}}} \ldots\left\|R^{n_{m+1}}\right\|_{p_{n_{m+1}}} \\
& =\sum_{n}\left\|A_{0}\right\| \ldots\left\|A_{m}\right\| \cdot\left\|R^{M+1}\right\|_{1} .
\end{aligned}
$$

Thus the iterated integral will be absolutely convergent if we show the boundedness of

$$
\begin{aligned}
& \int_{0}^{\infty} s^{M} \int_{-\infty}^{\infty}{\sqrt{a^{2}+v^{2}}}^{-q / 2-\operatorname{Re}(r)+(M-m) / 2}\left\|R^{M+1}\right\|_{1} d v d s \\
& \leq \int_{0}^{\infty} s^{M} \int_{-\infty}^{\infty}{\sqrt{a^{2}+v^{2}}}^{-q / 2-\operatorname{Re}(r)+(M-m) / 2}{\sqrt{\left(s^{2}+a\right)^{2}+v^{2}}}^{-M-1+q / 2+\varepsilon / 2} d v d s,
\end{aligned}
$$

the last inequality coming from Lemma 5.11 below and $\varepsilon>0$ is arbitrarily small. By Lemma 5.12 below, this integral is finite provided

$$
M-2(M+1-q / 2-\varepsilon / 2)<-1
$$

and

$$
M-2(M+1-q / 2-\varepsilon / 2)+1-2(q / 2+\operatorname{Re}(r)-(M-m) / 2)<-2 .
$$

These conditions reduce to

$$
q+\varepsilon<M+1, \quad(1-m) / 2<\operatorname{Re}(r)-\varepsilon / 2 .
$$

As $\varepsilon>0$ is arbitrary, we see that the integral is finite for $\operatorname{Re}(r)>(1-m) / 2$.

With a little more effort using the above estimate and the ideas in the proof of Lemma 5.4 in [CPRS2] one can show that the double integral is uniformly bounded in any closed right half-plane in the region $\operatorname{Re}(r)>(1-m) / 2$ and is $O(1 / \operatorname{Re}(r))$ as $\operatorname{Re}(r) \rightarrow \infty$. That is,

$$
\left|\phi_{m, t}^{r}\left(a_{0}, a_{1}, \ldots, a_{m}\right)\right| \leq C\left(r_{0}\right)\left\|a_{0}\right\| \cdot\left\|d a_{1}\right\| \ldots\left\|d a_{m}\right\|
$$

uniformly in $r$ with $\operatorname{Re}(r) \geq r_{0}>(1-m) / 2$. Moreover, $C\left(r_{0}\right)$ is $O\left(1 / r_{0}\right)$ as $r_{0} \rightarrow \infty$.

The fact that the map $\left.r \mapsto \phi_{m, t}^{r}\left(a_{0}, \ldots, a_{m}\right)\right)$ is holomorphic in the half-plane $\operatorname{Re}(r)>(1-m) / 2$ is similar to the proof of Lemma 7.4 of [CPRS2]. 
4.3. The residue cocycle. The semifinite local index formula in the form described above entails the introduction of the residue cocycle, which we now describe.

Definition 4.8. Let $(\mathcal{A}, \mathscr{H}, \mathscr{D})$ be a $Q C^{\infty}$ finitely summable spectral triple with isolated spectral dimension $q \geq 1$. Let $M=2 N-P$. For $m=P, P+2, \ldots, M$ and using the notation of Definition 4.2 define functionals $\phi_{m}$ by

$$
\begin{aligned}
\phi_{m}\left(a_{0}, \ldots, a_{m}\right)= & \sqrt{2 \pi i} \sum_{|k|=0}^{M-m}(-1)^{|k|} \alpha(k) \sum_{j=A}^{h} \sigma_{h, j} \tau_{j-A} \\
& \cdot\left(\gamma a_{0}\left[\mathscr{D}, a_{1}\right]^{\left(k_{1}\right)} \ldots\left[\mathscr{D}, a_{m}\right]^{\left(k_{m}\right)}\left(1+\mathscr{D}^{2}\right)^{-|k|-m / 2}\right),
\end{aligned}
$$

where $h=|k|+(m-P) / 2$. Here $\gamma$ denotes the $\mathbb{Z}_{2}$-grading in the even case and the identity operator in the odd case. Note that $M$ is the greatest odd (respectively, even) integer in $(q+1)$ when the spectral triple is odd (respectively, even).

It follows from the results of [CPRS2], [CPRS3] that $\phi=\left(\phi_{m}\right)_{m=P}^{M}$ is a $(b, B)$ cocycle, called the residue cocycle. The relationship between the resolvent cocycle and the residue cocycle is captured by the following result proved in [CPRS2], [CPRS3].

Theorem 4.9. Let $(\mathcal{A}, \mathscr{H}, \mathscr{D})$ be a $Q C^{\infty}$ finitely summable spectral triple with isolated spectral dimension $q \geq 1$. When evaluated on any $a_{0}, \ldots, a_{m} \in \mathcal{A}$, with $m \equiv P(\bmod 2)$ the components $\phi_{m}^{r}$ of the resolvent cocycle $\left(\phi^{r}\right)$ analytically continue to a deleted neighbourhood of $r=(1-q) / 2$. Moreover, if we denote this continuation by $\varphi_{m}^{r}\left(a_{0}, \ldots, a_{m}\right)$ then

$$
\operatorname{res}_{r=(1-q) / 2} \varphi_{m}^{r}\left(a_{0}, \ldots, a_{m}\right)=\phi_{m}\left(a_{0}, \ldots, a_{m}\right) .
$$

It is the proof of this last result that shows that the resolvent cocycle is indeed playing the same role as JLO does for the original proof of the local index theorem.

4.4. Statement of the theorem. Our main result on the residue cocycle establishes the following equality in cyclic cohomology.

Theorem 4.10. Let $(\mathcal{A}, \mathcal{H}, \mathscr{D})$ be a $Q C^{\infty}$ spectral triple (even or odd) with spectral dimension $q \geq 1$ and isolated spectral dimension. Then the residue cocycle represents the Chern character of the $K$-homology class of $(\mathcal{A}, \mathcal{H}, \mathfrak{D})$.

\section{The residue and resolvent cocycles represent the Chern character}

5.1. Preamble. Our methods are inspired by Higson $[\mathrm{H}]$ and we follow his approach quite closely. We present our arguments in full because numerous algebraic identities 
are, while very similar to Higson's, different in small details and the analytic estimates are very subtle. In fact numerous additional arguments are needed because we are working in much greater generality. Our immediate aim is to prove the following two statements and from these we will deduce our theorem on the residue cocycle.

Theorem 5.1. Let $(\mathcal{A}, \mathscr{H}, \mathcal{D})$ be a $Q C^{\infty}$ finitely summable spectral triple with dimension $q \geq 1$ and $D$ invertible. Let $M=2 N-P$ where $N=[(q+1+P) / 2]$, and note that in the odd (even) case, $M$ is the greatest odd (even) integer in $q+1$. Then in the $(b, B)$ bicomplex for $A$ with coefficients in functions holomorphic for $\operatorname{Re}(r)>1 / 2$, the resolvent cocycle $\left(\phi_{m}^{r}\right)_{m=P}^{M}$ is cohomologous to

$$
\frac{1}{(r-(1-q) / 2)} \mathrm{Ch}_{F}^{M}
$$

modulo cochains with values in the functions holomorphic in a half-plane containing $r=(1-q) / 2$. Here $F=\mathscr{D}|\mathscr{D}|^{-1}$ and $\mathrm{Ch}_{F}^{M}$ denotes the representative of the Chern character in dimension $M$.

Theorem 5.2. If $(\mathcal{A}, \mathcal{H}, \mathscr{D})$ is a $Q C^{\infty}$ spectral triple with isolated spectral dimension $q \geq 1$ and $\mathscr{D}$ invertible, then the cyclic cohomology class of the residue cocycle

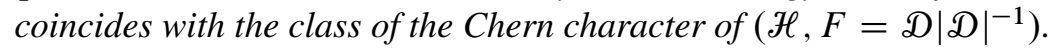

There are two main steps involved in proving these statements. First in Sections 5.3 and 5.4 we need to define a 'transgression' cochain which provides a cohomology (modulo cochains with values in the functions holomorphic in a half-plane containing $(1-q) / 2)$ between the resolvent cocycle and a single term cyclic cochain which is 'almost' a cocycle. Second in Section 5.5 we deform the resulting single term cyclic cochain to the Chern character. In this process we introduce error terms that are holomorphic at $r=(1-q) / 2$. Theorem 5.2 follows on taking residues at $(1-q) / 2$ and the isolated spectral dimension hypothesis enters at this stage. Both of these steps require invertibility of $\mathscr{D}$. However, once we have proved Theorems 5.1 and 5.2 in Section 5.6, we replace (in Section 5.7) $(\mathcal{A}, \mathscr{H}, \mathscr{D})$ by its double to remove this hypothesis.

The standing assumption for the rest of this section is that $(\mathcal{A}, \mathscr{H}, \mathscr{D})$ is a $Q C^{\infty}$ finitely summable spectral triple with dimension $q \geq 1$. We will at times assume isolated spectral dimension, but shall always be explicit in those results that need this hypothesis. Until we reach Section 5.7 we shall also assume that $\mathscr{D}$ is boundedly invertible.

5.2. Preliminary identities. We begin with some basic identities for the expectations $\langle\ldots\rangle_{m, r, s}$. 
Lemma 5.3. For $m \geq 0$ and operators $A_{0}, \ldots, A_{m}, A_{i} \in O P^{k_{i}}$, with $k_{0}+\cdots$ $\cdots+k_{m}-2 m-1<2 \operatorname{Re}(r)$, we have for $1 \leq j<m$ :

$$
\begin{aligned}
& -\left\langle A_{0}, \ldots,\left[\mathcal{D}^{2}, A_{j}\right], \ldots, A_{m}\right\rangle_{m, s, r} \\
& \quad=\left\langle A_{0}, \ldots, A_{j-1} A_{j}, \ldots, A_{m}\right\rangle_{m-1, s, r}-\left\langle A_{0}, \ldots, A_{j} A_{j+1}, \ldots, A_{m}\right\rangle_{m-1, s, r},
\end{aligned}
$$

while for $j=m$ we have

$$
\begin{aligned}
& -\left\langle A_{0}, \ldots, A_{m-1},\left[\mathscr{D}^{2}, A_{m}\right]\right\rangle_{m, s, r} \\
& \quad=\left\langle A_{0}, \ldots, A_{m-1} A_{m}\right\rangle_{m-1, s, r}-(-1)^{A \operatorname{deg}\left(A_{m}\right)}\left\langle A_{m} A_{0}, \ldots, A_{m-1}\right\rangle_{m-1, s, r} .
\end{aligned}
$$

For $k \geq 1$ we also have

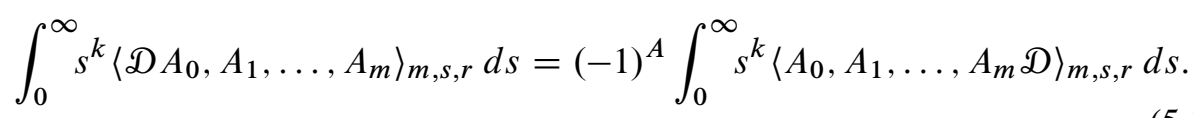

Moreover these s-integrals have a cyclic property:

$$
\int_{0}^{\infty} s^{k}\left\langle A_{0}, \ldots, A_{m}\right\rangle_{m, s, r} d s=(-1)^{A \operatorname{deg}\left(A_{m}\right)} \int_{0}^{\infty} s^{k}\left\langle A_{m}, A_{0}, \ldots, A_{m-1}\right\rangle_{m, s, r} d s .
$$

Furthermore if $\sum_{i=0}^{m} \operatorname{deg}\left(A_{i}\right) \equiv A(\bmod 2)$ and we define

$$
\operatorname{deg}_{-1}=0 \text { and } \operatorname{deg}_{k}=\operatorname{deg}\left(A_{0}\right)+\operatorname{deg}\left(A_{1}\right)+\cdots+\operatorname{deg}\left(A_{k}\right),
$$

then

$$
0=\sum_{j=0}^{m}(-1)^{\operatorname{deg}_{j-1}} \int_{0}^{\infty} s^{k}\left\langle A_{0}, \ldots,\left[\mathscr{D}, A_{j}\right]_{ \pm}, \ldots, A_{m}\right\rangle_{m, s, r} d s .
$$

Proof. The first half of the first statement is just the first statement of Lemma 7.8 of [CPRS2] in the odd case: the proof in the even case is the same. The second part of the first statement has a similar proof to the first part, but requires the factor $(-1)^{A \operatorname{deg}\left(A_{m}\right)}$ since $A_{m}$ may anticommute with $\gamma$ in the even case. The second statement is also the second statement of Lemma 7.8 of [CPRS2] in the odd case where $A=0$. The even case is the same argument but needs the factor $(-1)^{A}$ since $\mathscr{D}$ and $\gamma$ anticommute. Similar comments apply to the cyclic property, noting that $R_{S}(\lambda)$ has grading degree 0 in either case. The cyclic property in the odd case is Lemma 7.7 of [CPRS2].

The sum in (5.2) telescopes since $\left[\mathscr{D}, A_{j}\right]_{ \pm}=\mathscr{D} A_{j}-(-1)^{\operatorname{deg}\left(A_{j}\right)} A_{j} \mathscr{D}$ and $\operatorname{deg}_{j}=\operatorname{deg}_{j-1}+\operatorname{deg}\left(A_{j}\right)$. After telescoping the sum, one is left with equation (5.1) since $\sum_{i=0}^{m} \operatorname{deg}\left(A_{i}\right) \equiv A(\bmod 2)$.

To introduce the 'transgression cochain' we need a new expectation and some basic properties. 
Definition 5.4. For $m \geq 0$ and operators $A_{0}, \ldots, A_{m}, A_{i} \in O P^{k_{i}}$, with $k_{0}+\cdots$ $\cdots+k_{m}-2 m-1<2 \operatorname{Re}(r)$ define

$$
\begin{array}{r}
\left\langle\left\langle A_{0}, \ldots, A_{m}\right\rangle\right\rangle_{m, s, r}=\sum_{j=0}^{m}(-1)^{\operatorname{deg}_{j}}\left\langle A_{0}, \ldots, A_{j}, \mathcal{D}, A_{j+1}, \ldots, A_{m}\right\rangle_{m+1, s, r} \\
=\sum_{j=0}^{m}(-1)^{\operatorname{deg}_{j}} \tau\left(\frac{1}{2 \pi i} \gamma \int_{l} \lambda^{-q / 2-r} A_{0} R_{S}(\lambda) A_{1} \ldots\right. \\
\left.\ldots A_{j} R_{S}(\lambda) D R_{S}(\lambda) \ldots A_{m} R_{s}(\lambda) d \lambda\right) .
\end{array}
$$

We note that except for a factor of 2 and the possible \pm 1 factors this is just formal differentiation with respect to the "variable" $\mathscr{D}$.

Lemma 5.5. For $m \geq 0$ and operators $A_{0}, \ldots, A_{m}, A_{i} \in O P^{k_{i}}$, with $k_{0}+\cdots$ $\cdots+k_{m}-2 m-2<2 \operatorname{Re}(r)$ we have for $1 \leq j<m$ the identity:

$$
\begin{aligned}
& \text { - }\left\langle\left\langle A_{0}, \ldots,\left[\mathscr{D}^{2}, A_{j}\right], \ldots, A_{m}\right\rangle\right\rangle_{m, s, r} \\
& =\left\langle\left\langle A_{0}, \ldots, A_{j-1} A_{j}, \ldots, A_{m}\right\rangle\right\rangle_{m-1, s, r} \\
& -\left\langle\left\langle A_{0}, \ldots, A_{j} A_{j+1}, \ldots, A_{m}\right\rangle_{m-1, s, r}\right. \\
& +(-1)^{\operatorname{deg}_{j-1}}\left\langle A_{0}, \ldots,\left[D{ }^{2}, A_{j}\right]_{ \pm}, \ldots, A_{m}\right\rangle_{m, s, r},
\end{aligned}
$$

where we have a graded commutator in the last term. For $j=m$ we have the identity:

$$
\begin{aligned}
& -\left\langle\left\langle A_{0}, \ldots, A_{m-1},\left[D^{2}, A_{m}\right]\right\rangle_{m, s, r}\right. \\
& =\left\langle\left\langle A_{0}, \ldots, A_{m-1} A_{m}\right\rangle\right\rangle_{m-1, s, r}-(-1)^{P \operatorname{deg}\left(A_{m}\right)}\left\langle\left\langle A_{m} A_{0}, \ldots, A_{m-1}\right\rangle\right\rangle_{m-1, s, r} \\
& +(-1)^{\operatorname{deg}_{m-1}}\left\langle A_{0}, \ldots,\left[\mathscr{D}, A_{m}\right]_{ \pm}\right\rangle_{m, s, r} .
\end{aligned}
$$

If $\sum_{i=0}^{m} \operatorname{deg}\left(A_{i}\right) \equiv P(\bmod 2)$ and $\alpha \geq 1$ we also have the identity:

$$
\begin{aligned}
& \sum_{k=0}^{m}(-1)^{\operatorname{deg}_{k-1}} \int_{0}^{\infty} s^{\alpha}\left\langle\left\langle A_{0}, \ldots,\left[D, A_{k}\right]_{ \pm}, \ldots, A_{m}\right\rangle\right\rangle_{m, s, r} d s \\
& =\sum_{i=0}^{m} 2 \int_{0}^{\infty} s^{\alpha}\left\langle A_{0}, \ldots, A_{i}, D^{2}, \ldots, A_{m}\right\rangle_{m+1, s, r} d s .
\end{aligned}
$$

On the other hand, if $\sum_{i=0}^{m} \operatorname{deg}\left(A_{i}\right) \equiv A(\bmod 2)$ and $\alpha \geq 1$ then we have the following cyclic property for $\langle\langle\ldots\rangle\rangle$ :

$$
\int_{0}^{\infty} s^{\alpha}\left\langle\left\langle A_{0}, \ldots, A_{m}\right\rangle\right\rangle_{m, s, r} d s=(-1)^{P \operatorname{deg}\left(A_{m}\right)} \int_{0}^{\infty} s^{\alpha}\left\langle\left\langle A_{m}, A_{0}, \ldots, A_{m-1}\right\rangle\right\rangle_{m, s, r} d s .
$$


Proof. All statements are computations. The first two are careful applications of Lemma 5.3 and are easily checked by the reader for say, $m=2$. The third is as follows (we suppress the integrals $\int_{0}^{\infty} s^{\alpha} \ldots d s$ in order to lighten the notation):

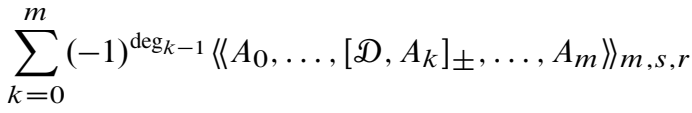

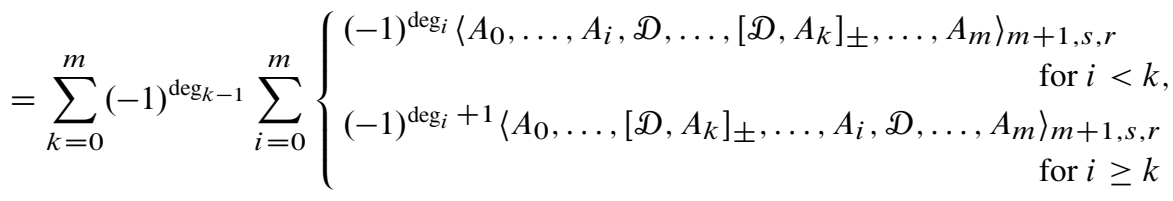

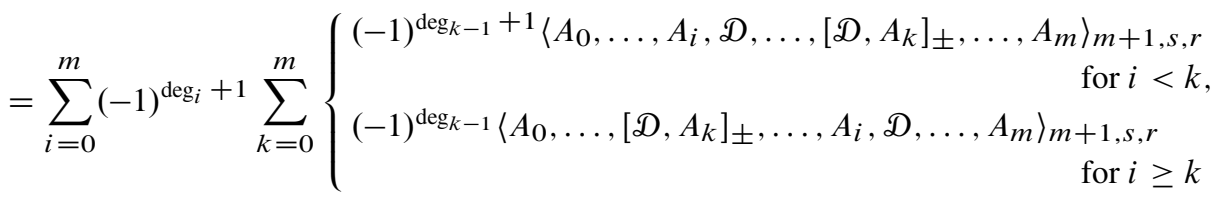

$$
\begin{aligned}
& +\sum_{i=0}^{m}(-1)^{\operatorname{deg}_{i}+1}\left((-1)^{\operatorname{deg}_{i}}\left\langle A_{0}, \ldots, A_{i}, 2 D^{2}, \ldots, A_{m}\right\rangle_{m+1, s, r}\right. \\
& \left.-(-1)^{\operatorname{deg}_{i}}\left\langle A_{0}, \ldots, A_{i}, 2 D^{2}, \ldots, A_{m}\right\rangle_{m+1, s, r}\right) \\
& =\sum_{i=0}^{m} 2\left\langle A_{0}, \ldots, A_{i}, D^{2}, \ldots, A_{m}\right\rangle_{m+1, s, r} .
\end{aligned}
$$

The last line follows by applying equation (5.2) above once for each $i=0, \ldots, m$ and noting that the graded commutator of $\mathscr{D}$ with itself is $2 \mathscr{D}^{2}$ and also that

$$
\sum_{j=0}^{m} \operatorname{deg}\left(A_{j}\right)+\operatorname{deg}(\mathscr{D}) \equiv A(\bmod 2) \Leftrightarrow \sum_{j=0}^{m} \operatorname{deg}\left(A_{j}\right) \equiv P(\bmod 2) .
$$

The fourth identity is a calculation using the cyclic property for $\langle\ldots\rangle$ in Lemma 5.3.

The next identity we refer to as the $s$-trick.

Lemma 5.6. For any integers $m \geq 0, \alpha \geq 1$ and operators $A_{0}, \ldots, A_{m}$ with $A_{j} \in O P^{k_{j}}, 1+\alpha+\sum k_{j}-2 m<2 \operatorname{Re}(r)$, we have

$$
\begin{aligned}
\alpha \int_{0}^{\infty} s^{\alpha-1}\left\langle A_{0}, \ldots, A_{m}\right\rangle_{m, s, r} d s & \\
& =-2 \sum_{j=0}^{m} \int_{0}^{\infty} s^{\alpha+1}\left\langle A_{0}, \ldots, A_{j}, 1, A_{j+1}, \ldots, A_{m}\right\rangle_{m+1, s, r} d s .
\end{aligned}
$$


Remark. Provided that $2 \operatorname{Re}(r)>\alpha+\sum k_{j}-2 m$, the $s$-trick works exactly the same for the expectation $\langle\langle\ldots\rangle\rangle$.

Proof. The derivative of $s^{\alpha}\left\langle A_{0}, \ldots, A_{m}\right\rangle_{m, s, r}$ is

$$
\alpha s^{\alpha-1}\left\langle A_{0}, \ldots, A_{m}\right\rangle_{m, s, r}+2 s^{\alpha+1} \sum_{i=0}^{m}\left\langle A_{0}, \ldots, A_{i}, 1, A_{i+1}, \ldots, A_{m}\right\rangle_{m+1, s, r} .
$$

Integrating this total derivative in $s$ from 0 to $\infty$ gives the result.

Similarly, we may employ our other variable of integration to obtain a $\lambda$-trick. The proof is virtually identical to the $s$-trick.

Lemma 5.7. For any integer $m \geq 0$, operators $A_{0}, \ldots, A_{m}$ with $A_{j} \in O P^{k_{j}}$ and $r$ such that $\sum k_{j}-2 m<2 \operatorname{Re}(r)$, we have

$$
-(q / 2+r)\left\langle A_{0}, \ldots, A_{m}\right\rangle_{m, s, r+1}=\sum_{j=0}^{m}\left\langle A_{0}, \ldots, A_{j}, 1, A_{j+1}, \ldots, A_{m}\right\rangle_{m+1, s, r} .
$$

Proof. We compute:

$$
\begin{aligned}
\frac{d}{d \lambda}\left(\lambda^{-(q / 2+r)} A_{0} R_{S}(\lambda) \ldots A_{m} R_{S}(\lambda)\right) & \\
= & -(q / 2+r) \lambda^{-(q / 2+r+1)} A_{0} R_{s}(\lambda) \ldots A_{m} R_{s}(\lambda) \\
& \quad-\lambda^{-(q / 2+r)} \sum_{j=0}^{m} A_{0} R_{S}(\lambda) \ldots A_{j} R_{S}(\lambda)^{2} A_{j+1} \ldots A_{m} R_{S}(\lambda) .
\end{aligned}
$$

Integrating this equation along the line $l$, multiplying by $\frac{\gamma}{2 \pi i}$ and taking the trace $\tau$ gives the result.

Lemma 5.8. For $m, \alpha \geq 0$, operators $A_{i} \in O P^{k_{i}}$ and $r$ such that $2 \operatorname{Re}(r)>1+\alpha-$ $2 m+\sum k_{j}$ we have

$$
\begin{aligned}
\sum_{j=0}^{m} \int_{0}^{\infty} s^{\alpha} & \left\langle A_{0}, \ldots, A_{j}, D^{2}, A_{j+1}, \ldots, A_{m}\right\rangle_{m+1, s, r} d s \\
= & -(m+1) \int_{0}^{\infty} s^{\alpha}\left\langle A_{0}, \ldots, A_{m}\right\rangle_{m, s, r} d s \\
& +(1-q / 2-r) \int_{0}^{\infty} s^{\alpha}\left\langle A_{0}, \ldots, A_{m}\right\rangle_{m, s, r} d s
\end{aligned}
$$




$$
\begin{aligned}
& +\frac{(\alpha+1)}{2} \int_{0}^{\infty} s^{\alpha}\left\langle A_{0}, \ldots, A_{m}\right\rangle_{m, s, r} d s \\
& -\sum_{j=0}^{m} \int_{0}^{\infty} s^{\alpha}\left\langle A_{0}, \ldots, A_{j}, 1, A_{j+1}, \ldots, A_{m}\right\rangle_{m+1, s, r} d s
\end{aligned}
$$

Proof. This uses $\mathscr{D}^{2} R_{S}(\lambda)=-1+\left(\lambda-\left(1+s^{2}\right)\right) R_{S}(\lambda)$. So

$$
\begin{aligned}
\left\langle A_{0}, \ldots, A_{j}, D^{2}, A_{j+1}, \ldots, A_{m}\right\rangle_{m+1, s, r} & \\
= & -\left\langle A_{0}, \ldots, A_{m}\right\rangle_{m, s, r}+\left\langle A_{0}, \ldots, A_{j}, 1, A_{j+1}, \ldots, A_{m}\right\rangle_{m+1, s, r-1} \\
& -\left(1+s^{2}\right)\left\langle A_{0}, \ldots, A_{j}, 1, A_{j+1}, \ldots, A_{m}\right\rangle_{m+1, s, r} .
\end{aligned}
$$

Summing gives us

$$
\begin{aligned}
\sum_{j=0}^{m}\left\langle A_{0}, \ldots, A_{j}, D^{2}, A_{j+1}, \ldots, A_{m}\right\rangle_{m+1, s, r} & \\
= & -(m+1)\left\langle A_{0}, \ldots, A_{m}\right\rangle_{m, r, s}+(1-q / 2-r)\left\langle A_{0}, \ldots, A_{m}\right\rangle_{m, s, r} \\
& -\sum_{j=0}^{m}\left(1+s^{2}\right)\left\langle A_{0}, \ldots, A_{j}, 1, A_{j+1}, \ldots, A_{m}\right\rangle_{m+1, s, r}
\end{aligned}
$$

here we used the $\lambda$-trick, Lemma 5.7. Now integrate over $s$ and use the $s$-trick (Lemma 5.6) on the $s^{2}$ part of the last term to obtain the result.

5.3. The first homotopy. We introduce a homotopy parameter into our resolvent cocycle and prove a transgression type formula.

Definition 5.9. Assume that $\mathscr{D}$ is invertible. For $t \in[0,1]$ define $R_{s, t}(\lambda)=$ $\left(\lambda-\left(t+s^{2}+\mathscr{D}^{2}\right)\right)^{-1}$ and let

$$
\langle\ldots\rangle_{m, r, s, t}, \quad\langle\ldots\rangle_{m, r, s, t}
$$

be the expectations of Definitions 4.5 and 5.4 using $R_{s, t}(\lambda)$ instead of $R_{s}(\lambda)$.

To see that this is well defined for $t \in[0,1]$, we must check that the trace estimates we require are still satisfied. These estimates all rest on the scalar inequality (see the proof of [CPRS2], Lemma 5.2)

$$
(X+Y)^{-a-b} \leq X^{-a} Y^{-b}
$$

for positive real numbers $X, Y, a, b$. For invertible $\mathscr{D}$ there exists a $\delta>0$ such that $\mathscr{D}^{2}-\delta \geq 0$. Therefore $\mathscr{D}^{-2},\left(\mathscr{D}^{2}-\delta / 2\right)^{-1}$ and $\left(1+\mathscr{D}^{2}\right)^{-1}$ all have the same 
summability (say, for any $K>q / 2$ ). So by the functional calculus if $K \geq q / 2+\varepsilon$ we have for all $t \in[0,1]$ :

$$
\begin{aligned}
\left(\mathscr{D}^{2}+t+s^{2}\right)^{-K} & =\left(\mathscr{D}^{2}-\delta / 2+\delta / 2+t+s^{2}\right)^{-K} \\
& \leq\left(\mathscr{D}^{2}-\delta / 2\right)^{-q / 2-\varepsilon}\left(\delta / 2+t+s^{2}\right)^{-K+q / 2+\varepsilon} \\
& \leq\left(\mathscr{D}^{2}-\delta / 2\right)^{-q / 2-\varepsilon}\left(\delta / 2+s^{2}\right)^{-K+q / 2+\varepsilon} .
\end{aligned}
$$

This is enough to prove part (b) of the following lemma. Part (a) is just the functional calculus. It is important to note that these lemmas imply that all the trace estimates will continue to hold uniformly for $t \in[0,1]$ provided that $\mathscr{D}$ is invertible. For our $\lambda$-integral we choose our line $l$ given by $\lambda=a+i v$ so that $0<a<\delta / 4$ to simply certain estimates, although this is not always needed.

In particular, we will need the following modifications of the results in [CPRS2].

Lemma 5.10 (Cf. [CPRS2], Lemma 5.1). Let $D$ be an unbounded self-adjoint operator with $\mathscr{D}^{2} \geq \delta$.

(a) For $\lambda=a+i v \in \mathbb{C}, 0<a<\delta / 4$, and $t, s \geq 0$ we have the estimate

$$
\left\|\left(\lambda-\left(t+D^{2}+s^{2}\right)\right)^{-1}\right\| \leq\left(v^{2}+\left(s^{2}+t+\delta-a\right)^{2}\right)^{-1 / 2} \leq \frac{1}{\delta-a} .
$$

(b) For $t, s \geq 0$ we have the estimate

$$
\left(\mathscr{D}^{2}+t+s^{2}\right)^{-K} \leq\left(D^{2}-\delta / 2\right)^{-q / 2-\varepsilon}\left(\delta / 2+s^{2}\right)^{-K+q / 2+\varepsilon} .
$$

Lemma 5.11 (Cf. [CPRS2], Lemma 5.3). Let $\mathscr{D}^{2} \geq \delta$. Let $\lambda=a+i v \in \mathbb{C}$, $0<a<\delta / 4$ and $t, s \geq 0$. For $q \geq 1$ let $\left(1+D^{2}\right)^{-1 / 2}$ be $(q+\varepsilon)$-summable for every $\varepsilon>0$. Then for each $\varepsilon>0$ and $N>(q+\varepsilon) / 2$, we have the trace norm estimate

$$
\begin{aligned}
\left\|\left(\lambda-\left(t+D^{2}+s^{2}\right)\right)^{-N}\right\|_{1} & \leq C_{q+\varepsilon}^{\prime}\left(\left(\delta / 2+s^{2}-a\right)^{2}+v^{2}\right)^{-N / 2+(q+\varepsilon) / 4} \\
& \leq C_{q+\varepsilon}^{\prime}\left(\left(s^{2}+a\right)^{2}+v^{2}\right)^{-N / 2+(q+\varepsilon) / 4},
\end{aligned}
$$

where $C_{q+\varepsilon}^{\prime}=\left\|\left(D^{2}-\delta / 2\right)^{-(q+\varepsilon) / 2}\right\|_{1}$.

Lemma 5.12 (Cf. [CPRS2], Lemma 5.4). Let $0<a<\delta / 4, t \in[0,1]$ and let $J, K, B \geq 0$. Then

$$
\begin{aligned}
& \int_{0}^{\infty} \int_{-\infty}^{\infty} s^{J}{\sqrt{a^{2}+v^{2}}}^{-B} \sqrt{\left(t+s^{2}+\delta / 2-a\right)^{2}+v^{2}}-K d v d s \\
& \leq \int_{0}^{\infty} \int_{-\infty}^{\infty} s^{J}{\sqrt{a^{2}+v^{2}}}^{-B}{\sqrt{\left(s^{2}+a\right)^{2}+v^{2}}}^{-K} d v d s
\end{aligned}
$$


converges provided that $J-2 K<-1$ and $J-2 K+1-2 B<-2$. Moreover, if $K$ and $J$ are fixed with $J-2 K<-1$, then the integral as a function of $B$ goes to 0 as $B \rightarrow \infty$. In particular, with $J, K$ fixed and $B_{0}$ some value for which the integral is finite, then the integrals are uniformly bounded for all $B \geq B_{0}$.

Definition 5.13. Define, for $\operatorname{Re}(r)>(1-m) / 2$, the components of the 'transgression' cochain

$$
\Phi_{m, t}^{r}\left(a_{0}, \ldots, a_{m}\right)=\frac{\eta_{m+1}}{2} \int_{0}^{\infty} s^{m+1}\left\langle\left\langle a_{0}, d a_{1}, \ldots, d a_{m}\right\rangle\right\rangle_{m, s, r, t} d s .
$$

Similarly, we define $\phi_{m, t}^{r}$ using $R_{s, t}(\lambda)$ in place of $R_{s}(\lambda)$ in the definition of $\phi^{r}$.

Remarks. Lemmas 5.3 to 5.7 inclusive work equally well for the expectations $\langle\ldots\rangle_{m, s, r, t}$ and $\langle\langle\ldots\rangle\rangle_{m, s, r, t}$, however Lemma 5.8 has an extra factor of $t$ on the last term as we will make explicit at the end of the proof of the following proposition.

Proposition 5.14. We have the $(b, B)$ bicomplex formula (with coefficients in the functions holomorphic for $\operatorname{Re}(r)>1 / 2)$ for $m \equiv P(\bmod 2)$

$$
\begin{aligned}
\left(B \Phi_{m+1, t}^{r}\right. & \left.+b \Phi_{m-1, t}^{r}\right)\left(a_{0}, \ldots, a_{m}\right) \\
& =\left(\frac{q-1}{2}+r\right) \phi_{m, t}^{r}\left(a_{0}, \ldots, a_{m}\right)-t \frac{q+2 r}{2} \phi_{m, t}^{r+1}\left(a_{0}, \ldots, a_{m}\right) .
\end{aligned}
$$

Proof. We begin the proof by computing with $\Phi_{m}^{r}:=\Phi_{m, 1}^{r}$. First, using the cyclic property of $\langle\langle\ldots\rangle\rangle$ of Lemma 5.5 and the fact that $m \equiv P(\bmod 2)$ we have writing $d a=[\mathscr{D}, a]:$

$$
\begin{aligned}
B \Phi_{m+1}^{r}\left(a_{0}, \ldots, a_{m}\right) & \\
& =\frac{\eta_{m+2}}{2} \sum_{j=0}^{m} \int_{0}^{\infty} s^{m+2}(-1)^{m j}\left\langle\left\langle 1, d a_{j}, \ldots, d a_{j-1}\right\rangle\right\rangle_{m+1, s, r} d s \\
& =\frac{\eta_{m+2}}{2} \sum_{j=0}^{m} \int_{0}^{\infty} s^{m+2}\left\langle\left\langle d a_{0}, \ldots, d a_{j-1}, 1, d a_{j}, \ldots, d a_{m}\right\rangle\right\rangle_{m+1, s, r} d s \\
& =-\frac{\eta_{m+2}(m+1)}{4} \int_{0}^{\infty} s^{m}\left\langle\left\langle d a_{0}, \ldots, d a_{m}\right\rangle\right\rangle_{m, s, r} d s \\
& =-\frac{\eta_{m}}{2} \int_{0}^{\infty} s^{m}\left\langle\left\langle d a_{0}, \ldots, d a_{m}\right\rangle\right\rangle_{m, s, r} d s .
\end{aligned}
$$

Here we use the $s$-trick (Lemma 5.6) in the last line, which is the same for $\langle\langle\ldots\rangle\rangle$ as for $\langle\ldots\rangle$. 
The computation for $b \Phi_{m-1}^{r}$ is the same as for $b \phi_{m-1}^{r}$ [CPRS2], except we need to take account of equation (5.3). This gives

$$
\begin{aligned}
b \Phi_{m-1}^{r} & \left(a_{0}, \ldots, a_{m}\right) \\
= & \frac{\eta_{m}}{2} \sum_{j=1}^{m}(-1)^{j} \int_{0}^{\infty} s^{m}\left\langle\left\langle a_{0}, d a_{1}, \ldots,\left[\mathscr{D}^{2}, a_{j}\right], \ldots, d a_{m}\right\rangle\right\rangle_{m, s, r} d s \\
& \quad-\frac{\eta_{m}}{2} \sum_{j=1}^{m} \int_{0}^{\infty} s^{m}\left\langle a_{0}, d a_{1}, \ldots, d a_{j}, \ldots, d a_{m}\right\rangle_{m, s, r} d s \\
= & \frac{\eta_{m}}{2} \sum_{j=1}^{m}(-1)^{j} \int_{0}^{\infty} s^{m}\left\langle\left\langle a_{0}, d a_{1} \ldots,\left[D^{2}, a_{j}\right], \ldots, d a_{m}\right\rangle\right\rangle_{m, s, r} d s \\
& -\frac{\eta_{m} m}{2} \int_{0}^{\infty} s^{m}\left\langle a_{0}, d a_{1}, \ldots, d a_{m}\right\rangle_{m, s, r} d s .
\end{aligned}
$$

Now put these together. First, using $\eta_{m+2}(m+1) / 2=\eta_{m}$ we obtain

$$
\begin{aligned}
\left(B \Phi_{m+1}^{r}\right. & \left.+b \Phi_{m-1}^{r}\right)\left(a_{0}, \ldots, a_{m}\right) \\
= & -\frac{\eta_{m}}{2} \int_{0}^{\infty} s^{m}\left\langle\left\langle d a_{0}, \ldots, d a_{m}\right\rangle\right\rangle_{m, s, r} d s \\
& +\frac{\eta_{m}}{2} \sum_{j=1}^{m}(-1)^{j} \int_{0}^{\infty} s^{m}\left\langle\left\langle a_{0}, d a_{1}, \ldots,\left[\mathscr{D}^{2}, a_{j}\right], \ldots, d a_{m}\right\rangle\right\rangle_{m, s, r} d s \\
& \quad-\frac{\eta_{m} m}{2} \int_{0}^{\infty} s^{m}\left\langle a_{0}, d a_{1}, \ldots, d a_{m}\right\rangle_{m, s, r} d s,
\end{aligned}
$$

then using the fact that $\left[\mathscr{D}^{2}, a_{j}\right]=\left[\mathscr{D},\left[\mathscr{D}, a_{j}\right]\right]_{ \pm}$we obtain

$$
\begin{aligned}
& =-\frac{\eta_{m}}{2}(-1)^{\operatorname{deg}\left(a_{0}\right)} \int_{0}^{\infty} s^{m}\left\langle\left[\left[\mathcal{D}, a_{0}\right]_{ \pm}, d a_{1}, \ldots, d a_{m}\right\rangle\right\rangle_{m, s, r} d s \\
& +\frac{-\eta_{m}}{2} \sum_{j=1}^{m}(-1)^{\operatorname{deg}\left(a_{0}\right)+\operatorname{deg}\left(d a_{1}\right)+\cdots+\operatorname{deg}\left(d a_{j-1}\right)} \\
& \cdot \int_{0}^{\infty} s^{m}\left\langle\left\langle a_{0}, d a_{1} \ldots,\left[\mathscr{D}, d a_{j}\right]_{ \pm}, \ldots, d a_{m}\right\rangle_{m, s, r} d s\right. \\
& -\frac{\eta_{m} m}{2} \int_{0}^{\infty} s^{m}\left\langle a_{0}, d a_{1}, \ldots, d a_{m}\right\rangle_{m, s, r} d s .
\end{aligned}
$$


Now, by identity (5.4) of Lemma 5.5, this gives

$$
\begin{aligned}
\left(B \Phi_{m+1}^{r}+b \Phi_{m-1}^{r}\right)\left(a_{0}, \ldots, a_{m}\right) & \\
= & \frac{-2 \eta_{m}}{2} \sum_{j=0}^{m} \int_{0}^{\infty} s^{m}\left\langle a_{0}, \ldots, d a_{j}, \mathcal{D}^{2}, d a_{j+1}, \ldots, d a_{m}\right\rangle_{m+1, s, r} d s \\
& \quad-\frac{\eta_{m} m}{2} \int_{0}^{\infty} s^{m}\left\langle a_{0}, d a_{1}, \ldots, d a_{m}\right\rangle_{m, s, r} d s,
\end{aligned}
$$

then applying Lemma 5.8 we obtain

$$
\begin{aligned}
= & -\eta_{m}\left(\left(-(m+1) \int_{0}^{\infty} s^{m}\left\langle a_{0}, d a_{1}, \ldots, d a_{m}\right\rangle_{m, s, r} d s\right.\right. \\
& +(1-q / 2-r) \int_{0}^{\infty} s^{m}\left\langle a_{0}, d a_{1}, \ldots, d a\right\rangle_{m, s, r} d s \\
& +\frac{(m+1)}{2} \int_{0}^{\infty} s^{m}\left\langle a_{0}, d a_{1}, \ldots, d a_{m}\right\rangle_{m, s, r} d s \\
& \left.-\sum_{j=0}^{m} \int_{0}^{\infty} s^{m}\left\langle a_{0}, \ldots, d a_{j}, 1, d a_{j+1}, \ldots, d a_{m}\right\rangle_{m+1, s, r} d s\right) \\
& -\frac{\eta_{m} m}{2} \int_{0}^{\infty} s^{m}\left\langle a_{0}, d a_{1}, \ldots, d a_{m}\right\rangle_{m, s, r} d s \\
= & \eta_{m} \frac{q+2 r-1}{2} \int_{0}^{\infty} s^{m}\left\langle a_{0}, d a_{1}, \ldots, d a_{m}\right\rangle_{m, s, r} d s \\
& +\eta_{m} \sum_{j=0}^{m} \int_{0}^{\infty} s^{m}\left\langle a_{0}, \ldots, d a_{j}, 1, d a_{j+1}, \ldots, d a_{m}\right\rangle_{m+1, s, r} d s \\
= & \frac{q}{+2 r-1} \phi_{m}^{r}\left(a_{0}, \ldots, a_{m}\right)-\frac{q+2 r}{2} \phi_{m}^{r+1}\left(a_{0}, \ldots, a_{m}\right) .
\end{aligned}
$$

We used the $\lambda$-trick (Lemma 5.7) in the last line.

We now do the general case of $t \in[0,1]$ and observe that for $\alpha \geq 1$, a slight variation on Lemma 5.8 gives

$$
\begin{aligned}
\sum_{j=0}^{m} \int_{0}^{\infty} s^{\alpha}\left\langle A_{0}, \ldots, A_{j}, D^{2}, A_{j+1}, \ldots, A_{m}\right\rangle_{m+1, r, s, t} d s \\
=-(m+1) \int_{0}^{\infty} s^{\alpha}\left\langle A_{0}, \ldots, A_{m}\right\rangle_{m, r, s, t} d s \\
\quad+(1-q / 2-r) \int_{0}^{\infty} s^{\alpha}\left\langle A_{0}, \ldots, A_{m}\right\rangle_{m, r, s, t} d s
\end{aligned}
$$




$$
\begin{aligned}
& +\frac{(\alpha+1)}{2} \int_{0}^{\infty} s^{\alpha}\left\langle A_{0}, \ldots, A_{m}\right\rangle_{m, r, s, t} d s \\
& -t \sum_{j=0}^{m} \int_{0}^{\infty} s^{\alpha}\left\langle A_{0}, \ldots, A_{j}, 1, A_{j+1}, \ldots, A_{m}\right\rangle_{m+1, r, s, t} d s .
\end{aligned}
$$

As mentioned in the remark, the use of Lemmas 5.3 to 5.7 in the proof for $t=1$ work equally well for $t \neq 1$ and now Lemma 5.8 is modified as above. Putting these results together with the computation in the case $t=1$ gives us:

$$
\begin{aligned}
\left(B \Phi_{m+1, t}^{r}+b \Phi_{m-1, t}^{r}\right)\left(a_{0}, \ldots, a_{m}\right) & \\
= & \left(\frac{q+2 r-1}{2}\right) \phi_{m, t}^{r}\left(a_{0}, \ldots, a_{m}\right) \\
& \quad+t \eta_{m} \sum_{j=0}^{m} \int_{0}^{\infty} s^{m}\left\langle a_{0}, \ldots, d a_{j}, 1, d a_{j+1}, \ldots, d a_{m}\right\rangle_{m+1, r, s, t} d s \\
& =\left(\frac{q+2 r-1}{2}\right) \phi_{m, t}^{r}\left(a_{0}, \ldots, a_{m}\right)-t \frac{q+2 r}{2} \phi_{m, t}^{r+1}\left(a_{0}, \ldots, a_{m}\right) .
\end{aligned}
$$

Corollary 5.15. For $\mathcal{D}$ invertible and $m \equiv P(\bmod 2)($ setting $t=0)$ we have

$$
\left(B \Phi_{m+1,0}^{r}+b \Phi_{m-1,0}^{r}\right)\left(a_{0}, \ldots, a_{m}\right)=\left(\frac{q-1}{2}+r\right) \phi_{m, 0}^{r}\left(a_{0}, \ldots, a_{m}\right) .
$$

Proposition 5.16. For D invertible, the cochain $\left(\phi_{m, 0}^{r}\right)_{m=P}^{\infty}$ is cohomologous to the cochain

$$
\frac{1}{(r-(1-q) / 2)} B \Phi_{M+1,0}^{r} .
$$

This cochain is a cyclic cocycle modulo cochains with values in the functions holomorphic at $r=(1-q) / 2$.

Proof. Let $M=2 N-P$. By Proposition 5.15 the cochain given by the infinite tuple

$$
\left(\frac{1}{(r+(q-1) / 2)} \Phi_{P-1,0}^{r}, \ldots, \frac{1}{(r+(q-1) / 2)} \Phi_{M-1,0}^{r}, 0,0, \ldots\right)
$$

$(B, b)$-cobounds the following difference. That is, applying $(B, b)$ to the above cochain yields

$$
\left(\phi_{P, 0}^{r}, \phi_{P+2,0}^{r}, \ldots, \phi_{M, 0}^{r}-\frac{B \Phi_{M+1,0}^{r}}{(r+(1-q) / 2)}, 0,0, \ldots\right)=\left(\left(\phi_{m, 0}^{r}\right)_{m=P}^{M}-\frac{B \Phi_{M+1,0}^{r}}{(r+(q-1) / 2)}\right) .
$$

That is, $\left(\phi_{m, 0}^{r}\right)$ is cohomologous to $\frac{1}{(r+(q-1) / 2)} B \Phi_{M+1,0}^{r}$. Observe that because it is in the image of $B,(r-(1-q) / 2)^{-1} B \Phi_{M+1,0}^{r}$ is cyclic. It is also a $b$-cyclic cocycle modulo cochains with values in the functions holomorphic at $r=(1-q) / 2$. This follows from

$$
b \Phi_{M-1,0}^{r}+B \Phi_{M+1,0}^{r}=(r+(q-1) / 2) \phi_{M, 0}^{r}
$$

by applying $b$ and recalling that $b \phi_{M, 0}^{r}$ is holomorphic at $r=(1-q) / 2$ [CPRS2]. 
Corollary 5.17. For $\mathscr{D}$ invertible and assuming that $(\mathcal{A}, \mathscr{H}, \mathscr{D})$ has isolated spectral dimension, the residue cocycle is cohomologous to $B \Phi_{M+1,0}^{(1-q) / 2}$. Moreover $B \Phi_{M+1,0}^{(1-q) / 2}$ is a cyclic cocycle.

Proof. By Proposition 5.15 we have that

$$
\left(\operatorname{res}_{r=(1-q) / 2} \frac{1}{(r+(q-1) / 2)} \Phi_{P-1,0}^{r}, \ldots, \operatorname{res}_{r=(1-q) / 2} \frac{1}{(r+(q-1) / 2)} \Phi_{M-1,0}^{r}, 0,0, \ldots\right)
$$

$(B, b)$-cobounds the difference

$$
\begin{aligned}
& \operatorname{res}_{r=(1-q) / 2}(\left.\phi_{P, 0}^{r}, \phi_{P+2,0}^{r}, \ldots, \phi_{M, 0}^{r}-\frac{B \Phi_{M+1,0}^{r}}{(r+(1-q) / 2)}, 0,0, \ldots\right) \\
&=\left(\phi_{P, 0}, \phi_{P+2,0}, \ldots, \phi_{M, 0}-B \Phi_{M+1,0}^{(1-q) / 2}, 0,0, \ldots\right) .
\end{aligned}
$$

This statement requires that $b \Phi_{M-1,0}^{r}\left(a_{0}, \ldots, a_{M}\right)$ is holomorphic at $r=(1-q) / 2$ and that $B \Phi_{M+1,0}^{r}$ is finite (and so holomorphic) at $r=(1-q) / 2$. This is easy to prove using [CPRS2], Lemmas 7.1, 7.2. Finally, to see that $B \Phi_{M+1,0}^{(1-q) / 2}$ is a cyclic cocycle, we simply take residues of the corresponding result in Proposition 5.16.

5.4. The homotopy to $\boldsymbol{t}=\mathbf{0}$. In what follows we suppose that $t, t+\varepsilon \in[0,1]$ and write as usual $R_{s, t}(\lambda)=\left(\lambda-\left(t+s^{2}+\mathscr{D}^{2}\right)\right)^{-1}$. By the resolvent equation $R_{s, t+\varepsilon}(\lambda)-R_{s, t}(\lambda)=\varepsilon R_{s, t+\varepsilon}(\lambda) R_{s, t}(\lambda)$ and the fact that $\left|R_{s, t}(\lambda)\right| \leq\left|R_{s, 0}(\lambda)\right|$ we see that

$$
\left\|R_{s, t+\varepsilon}(\lambda)-R_{s, t}(\lambda)\right\|_{M+1} \leq \frac{\varepsilon}{\delta-a}\left\|R_{s, 0}(\lambda)\right\|_{M+1} .
$$

Similar considerations show that:

$$
\left\|\frac{1}{\varepsilon}\left(R_{S, t+\varepsilon}(\lambda)-R_{s, t}(\lambda)\right)-R_{S, t}(\lambda)^{2}\right\|_{M+1} \leq \frac{\varepsilon}{(\delta-a)^{2}}\left\|R_{s, 0}(\lambda)\right\|_{M+1} .
$$

At this point we lighten the notation temporarily by dropping the dependence on $\lambda$. More generally, if $n \geq 1$, then we can make use of the identity $X^{n}-Y^{n}=$ $\sum_{k=0}^{n-1} X^{k}(X-Y) Y^{n-k-1}$ and of Hölder's inequality to show that

and

$$
\left\|R_{s, t+\varepsilon}^{n}-R_{s, t}^{n}\right\|_{\frac{M+1}{n}} \leq \frac{\varepsilon \cdot n}{\delta-a}\left\|R_{s, 0}\right\|_{M+1}^{n}
$$

$$
\left\|\frac{1}{\varepsilon}\left(R_{s, t+\varepsilon}^{n}-R_{s, t}^{n}\right)-n R_{s, t}^{n+1}\right\|_{\frac{M+1}{n}} \leq \frac{\varepsilon}{(\delta-a)^{2}} \cdot \frac{n(n+1)}{2}\left\|R_{s, 0}\right\|_{M+1}^{n} .
$$

Let $F_{m}$ be the space of functions defined and holomorphic in the right half-plane $\{z \in \mathbb{C} \mid \operatorname{Re}(z)>(1-m) / 2\}$, and give $F_{m}$ the topology of uniform convergence on compacta. 
Proposition 5.18. For each $m=P, P+2, \ldots$ the map $[0,1] \ni t \mapsto \phi_{m, t}^{\bullet} \in$ $H\left(\mathcal{A}^{\otimes m+1}, F_{m}\right)$ is $C^{1}$ and

$$
\frac{d}{d t} \phi_{m, t}^{\bullet}=-(q / 2+\bullet) \phi_{m, t}^{\bullet+1}
$$

Proof. We do the case $m<M$ where we must use some initial trickery to get to a computable situation. For $m \geq M$ such tricks are not needed. We recall from the proof of Lemma 4.7 that if we apply the $s$-trick $(M-m) / 2$ times and the $\lambda$-trick $(M-m) / 2$ times we obtain by summing over all $k=\left(k_{0}, \ldots, k_{m}\right)$ where each $0 \leq k_{i} \leq(M-m)$ and $|k|=(M-m)$ :

$$
\begin{aligned}
\int_{0}^{\infty} s^{m}\left\langle a_{0}, d a_{1}, \ldots, d a_{m}\right\rangle_{m, s, r, t} d s \\
=(2)^{\frac{M-m}{2}}(M-m) ! \prod_{l=1}^{\frac{M-m}{2}} \frac{1}{q / 2+r-l} \prod_{j=1}^{\frac{M-m}{2}} \frac{1}{m+j} \\
\quad \cdot \sum_{k} \int_{0}^{\infty} s^{M}\left\langle a_{0}, 1^{k_{0}}, d a_{1}, 1^{k_{1}}, \ldots, d a_{m}, 1^{k_{m}}\right\rangle_{M, s, r-(M-m) / 2, t} d s .
\end{aligned}
$$

Where, we mean $1^{k_{i}}=1,1, \ldots, 1$ with $k_{i}$ one's. Ignoring the prefactors on the right-hand side, this becomes

$$
\sum_{n} \int_{0}^{\infty} s^{M} \tau\left(\frac{1}{2 \pi i} \gamma \int_{l} \lambda^{-q / 2-r+(M-m) / 2} a_{0} R_{s, t}^{n_{0}} d a_{1} R_{s, t}^{n_{1}} \ldots d a_{m} R_{s, t}^{n_{m}} d \lambda\right) d s
$$

where we sum over $n=\left(n_{0}, \ldots, n_{m}\right)$ with each $n_{i}=k_{i}+1$ so that $1 \leq n_{i} \leq$ $(M-m)+1$ and $|n|=M+1$. Now each integrand is not only trace class, but by the estimates immediately preceding the statement of this proposition they are $t$-differentiable in trace norm using the usual product rule argument and Hölder's inequality. In particular we have

$$
\begin{aligned}
& \| \frac{1}{\varepsilon}\left(a_{0} R_{s, t+\varepsilon}^{n_{0}} d a_{1} \ldots d a_{m} R_{s, t+\varepsilon}^{n_{m}}-a_{0} R_{s, t}^{n_{0}} d a_{1} \ldots d a_{m} R_{s, t}^{n_{m}}\right) \\
& \quad-\sum_{i=0}^{m} n_{i} a_{0} R_{s, t}^{n_{0}} \ldots d a_{i} R_{s, t}^{n_{i}+1} \ldots d a_{m} R_{s, t}^{n_{m}} \|_{1} \\
& \leq C \varepsilon\left\|a_{0}\right\| \cdot\left\|d a_{1}\right\| \ldots\left\|d a_{m}\right\| \cdot\left\|R_{s, 0}\right\|_{M+1},
\end{aligned}
$$

where the constant $C$ is independent of $s, \lambda$ and $r$. Since

$$
\int_{0}^{\infty} s^{M} \int_{l}\left|\lambda^{-q / 2-r+(M-m) / 2}\right| \cdot\left\|R_{s, 0}(\lambda)\right\|_{M+1} d \lambda d s=O\left(\operatorname{Re}(r)^{-1}\right)<\infty
$$


by Lemma 5.12, we can invoke the Lebesgue Convergence Theorem to conclude that $d / d t\left(\phi_{m, t}^{r}\left(a_{0}, \ldots, a_{m}\right)\right)$ exists and equals

$$
\begin{gathered}
\eta_{m} 2^{\frac{M-m}{2}}(M-m) ! \prod_{b=1}^{\frac{M-m}{2}} \frac{1}{q / 2+r-b} \prod_{j=1}^{\frac{M-m}{2}} \frac{1}{m+j} \sum_{k} \sum_{i=0}^{m} \int_{0}^{\infty} s^{M}\left(k_{i}+1\right) \\
\cdot\left\langle a_{0}, 1^{k_{0}}, \ldots, d a_{i}, 1^{k_{i}+1}, \ldots, d a_{m}, 1^{k_{m}}\right\rangle_{M+1, s, r-(M-m) / 2, t} d s .
\end{gathered}
$$

Now undoing our applications of the $s$-trick and the $\lambda$-trick we obtain

$$
\frac{d}{d t} \phi_{m, t}^{r}\left(a_{0}, \ldots, a_{m}\right)=\eta_{m} \sum_{j=0}^{m} \int_{0}^{\infty} s^{m}\left\langle a_{0}, \ldots, d a_{j}, 1, d a_{j+1}, \ldots, d a_{m}\right\rangle_{m+1, s, r, t} d s,
$$

and a final application of the $\lambda$-trick yields that

$$
\frac{d}{d t} \phi_{m, t}^{r}\left(a_{0}, \ldots, a_{m}\right)=-(q / 2+r) \phi_{m, t}^{r+1}\left(a_{0}, \ldots, a_{m}\right) .
$$

We note that by our estimates the convergence is uniform in $r$ for $r$ in a compact set.

In the discussion below let $k=0,1,2, \ldots$, let $m=P, P+2, \ldots, M$ and let $t \in[0,1]$.

Lemma 5.19. We have

$$
\phi_{m, t}^{r+k}=\frac{1}{r+k+(q-1) / 2}\left[B \Phi_{m+1, t}^{r+k}+b \Phi_{m-1, t}^{r+k}+(q / 2+r+k) t \phi_{m, t}^{r+k+1}\right] .
$$

Proof. This is just Proposition 5.14 with $r+k$ in place of $r$.

Proposition 5.20. For all $R, T \in[0,1]$, the cocycles $\left(\phi_{m, T}^{r}\right)_{m=P}^{M}$ and $\left(\phi_{m, R}^{r}\right)_{m=P}^{M}$ are equal modulo coboundaries and modulo cochains yielding functions holomorphic at $r=(1-q) / 2$.

Proof. Recall from Proposition 5.18 that for $\mathcal{D}$ invertible, $\phi_{m, t}^{r}$ is defined and holomorphic for $\operatorname{Re}(r)>(1-m) / 2$ for all $t \in[0,1]$. As $[0,1]$ is compact, the integral

$$
\int_{0}^{1} \phi_{m, t}^{r}\left(a_{0}, \ldots, a_{m}\right) d t
$$

is holomorphic for $\operatorname{Re}(r)>(1-m) / 2$ and any $a^{0}, \ldots, a^{m} \in \mathcal{A}$. 
Now we make some simple observations, omitting the variables $a_{0}, \ldots, a_{m}$ to lighten the notation. For $T, R \in[0,1]$ we have

$$
\phi_{m, T}^{r}-\phi_{m, R}^{r}=\int_{R}^{T} \frac{d}{d t} \phi_{m, t}^{r} d t=-(q / 2+r) \int_{R}^{T} \phi_{m, t}^{r+1} d t .
$$

Now apply the formula of Lemma 5.19 iteratively. First we obtain

$\phi_{m, T}^{r}-\phi_{m, R}^{r}=\frac{-(q / 2+r)}{r+1+(q-1) / 2} \int_{R}^{T}\left(B \Phi_{m+1, t}^{r+1}+b \Phi_{m-1, t}^{r+1}+(q / 2+r+1) t \phi_{m, t}^{r+2}\right) d t$.

Observe that the numerical factors are holomorphic at $r=(1-q) / 2$. Iterating this procedure $L$ times gives us

$$
\begin{aligned}
& \phi_{m, T}^{r}-\phi_{m, R}^{r}=\frac{-(q / 2+r) \ldots(q / 2+r+L)}{(r+1+(q-1) / 2) \ldots(r+L+(q-1) / 2)} \int_{R}^{T} t^{L} \phi_{m, t}^{r+L+1} d t \\
&+\sum_{j=1}^{L} \frac{-(q / 2+r) \ldots(q / 2+r+j-1)}{(r+1+(q-1) / 2) \ldots(r+j+(q-1) / 2)} \\
& \cdot \int_{R}^{T}\left(B \Phi_{m+1, t}^{r+j}+b \Phi_{m-1, t}^{r+j}\right) t^{j-1} d t .
\end{aligned}
$$

We would like to know (for completeness) what is the smallest integer $L$ that guarantees that $\phi_{m, t}^{r+L+1}$ is holomorphic at $r=(1-q) / 2$ for all $m$. We know that we require

$$
\operatorname{Re}(r)+L+1>(1-m) / 2
$$

for each $m$. Rearranging gives $\operatorname{Re}(r)>-1-L+(1-m) / 2$, and we would like the right-hand side to be strictly less than $(1-q) / 2$ for each $m$. In the even case the worst situation is when $m=0$, and for this case we require $L>-1+q / 2$. Since in the even case $N=[(q+1) / 2]>(q-1) / 2>-1+q / 2$, we may choose $L=N$. In the odd case the worst case is $m=1$, and we require $L>-1+(q-1) / 2$. In this case $N=[(q+2) / 2]>q / 2>(q-1) / 2$, and so we may take $L=N-1$. (If $N-1=0$, then we need do no iterations because equation (5.6) shows that modulo cochains yielding holomorphic functions, $\phi_{1, T}^{r}=\phi_{1, R}^{r}$, and these are the only functionals in the resolvent cocycle.)

With this choice of $L=N-P$, we have modulo cochains yielding functions holomorphic in a half-plane containing $(1-q) / 2$,

$$
\begin{gathered}
\phi_{m, T}^{r}-\phi_{m, R}^{r}=\sum_{j=1}^{L} \frac{-(q / 2+r) \ldots(q / 2+r+j-1)}{(r+1+(q-1) / 2 \ldots(r+j+(q-1) / 2)} \\
\cdot \int_{R}^{T}\left(B \Phi_{m+1, t}^{r+j}+b \Phi_{m-1, t}^{r+j}\right) t^{j-1} d t .
\end{gathered}
$$


Thus a simple rearrangement yields the cohomology, valid for $\operatorname{Re}(r)>(1-P) / 2$,

$$
\begin{gathered}
\left(\phi_{m, T}^{r}-\phi_{m, R}^{r}\right)_{m=P}^{M}-B \sum_{j=1}^{L} \frac{-(q / 2+r) \ldots(q / 2+r+j-1)}{(r+1+(q-1) / 2 \ldots(r+j+(q-1) / 2)} \\
\cdot \int_{R}^{T} \Phi_{M+1, t}^{r+j} t^{j-1} d t \\
=(B+b)\left(\sum_{j=1}^{L} \frac{-(q / 2+r) \ldots(q / 2+r+j-1)}{(r+1+(q-1) / 2 \ldots(r+j+(q-1) / 2)}\right. \\
\left.\cdot \int_{R}^{T} \Phi_{m, t}^{r+j} t^{j-1} d t\right)_{m=P}^{M-1} .
\end{gathered}
$$

Hence modulo coboundaries and cochains yielding functions holomorphic at $r=$ $(1-q) / 2$, we have the equality

$$
\begin{aligned}
& \left(\phi_{m, T}^{r}-\phi_{m, R}^{r}\right)_{m=P}^{M} \\
& \quad=B \sum_{j=1}^{L} \frac{-(q / 2+r) \ldots(q / 2+r+j-1)}{(r+1+(q-1) / 2 \ldots(r+j+(q-1) / 2)} \int_{R}^{T} \Phi_{M+1, t}^{r+j} t^{j-1} d t .
\end{aligned}
$$

However, an application of [CPRS2, Lemma 7.2] now shows that the right-hand side is holomorphic at $r=(1-q) / 2$, since $j \geq 1$ in all cases. Hence, modulo coboundaries and cochains yielding functions holomorphic at $r=(1-q) / 2$, we have the equality

$$
\left(\phi_{m, T}^{r}\right)_{m=P}^{M}=\left(\phi_{m, R}^{r}\right)_{m=P}^{M} .
$$

Corollary 5.21. Modulo coboundaries and cochains yielding functions holomorphic in a half-plane containing $r=(1-q) / 2$, we have the equality

$$
\left(\phi_{m}^{r}\right)_{m=P}^{M}=:\left(\phi_{m, 1}^{r}\right)_{m=P}^{M}=B \Phi_{M+1,0}^{r} .
$$

5.5. Homotopy to the Chern character for invertible $\mathfrak{D}$. We now drop the 0 subscript from $\Phi_{M+1,0}^{r}$ as we will leave $t=0$ from now on and consider a different homotopy. We now want to deform $B \Phi_{M+1}^{r}$ using the homotopy $u \rightarrow \mathscr{D}_{u}:=$ $\mathscr{D}|\mathscr{D}|^{-u}$, following the strategy of Higson. Unfortunately we discovered that this homotopy is quite subtle if one wants to check all of the estimates needed to show it is well defined. Hence the somewhat lengthy discussion in this section. Of course it is clear that this homotopy only makes sense in the invertible case. We handle the transition to the non-invertible case later.

We write $\Phi_{u, M+1}^{r}$ for $\Phi_{M+1}^{r}$ defined using $\mathscr{D}_{u}$ instead of $\mathscr{D}=\mathscr{D}_{0}$. We would also like to write $\dot{D}=-\mathscr{D}_{u} \log |\mathscr{D}|$, but this is purely formal and we will only take the limit of the corresponding difference quotients when they are multiplied by a 
factor of $|\mathscr{D}|^{-\rho}$ to ensure that the limit exists. In fact this derivative only appears via the derivative of $B \Phi_{u, M+1}^{r}$, which in turn appears in the coboundary computation of yet another auxiliary cochain $\Psi_{u, M}^{r}$ in Lemma 5.22 below. The necessary estimates for taking the derivative of $B \Phi_{u, M+1}^{r}$ are proved in Lemma 5.23.

Lemma 5.22. For $r>(1-M) / 2$ define a functional by

$$
\Psi_{u, M}^{r}\left(a_{0}, \ldots, a_{M}\right)=-\frac{\eta_{M}}{2} \int_{0}^{\infty} s^{M}\left\langle\left\langle a_{0} \dot{\mathscr{D}}_{u},\left[\mathscr{D}_{u}, a_{1}\right], \ldots,\left[\mathscr{D}_{u}, a_{M}\right]\right\rangle\right\rangle_{M, s, r} d s
$$

Then we have

$$
\begin{aligned}
& \left(b B \Psi_{u, M}^{r}\right)\left(a_{0}, \ldots, a_{M}\right) \\
& =-\eta_{M}(r+(q-1) / 2) \sum_{i=0}^{M}(-1)^{i} \\
& \quad \cdot \int_{0}^{\infty} s^{M}\left\langle\left[\mathscr{D}_{u}, a_{0}\right], \ldots,\left[\mathscr{D}_{u}, a_{i}\right], \dot{D}_{u}, \ldots,\left[\mathscr{D}_{u}, a_{M}\right]\right\rangle_{M+1, s, r} d s \\
& \quad+\frac{d}{d u}\left(B \Phi_{u, M+1}^{r}\right)\left(a_{0}, \ldots, a_{M}\right) .
\end{aligned}
$$

Remarks. (i) A judicious use of Hölder's inequality shows directly that $\Psi_{u, M}^{r}$ is finite for $\operatorname{Re}(r)>(1-M) / 2$. However we choose a slightly different argument and obtain this fact as a corollary of the computations below .

(ii) The derivative of $B \Phi_{u, M+1}^{r}$ must be shown to exist for $\operatorname{Re}(r)>(1-M) / 2$. The functional $B \Phi_{u, M+1}^{r}$ is finite in this region, and so a similar argument to that which shows $\phi_{m}^{r}$ is holomorphic when finite will show that $B \Phi_{u, M+1}^{r}$ is holomorphic when finite. In fact $B \Phi_{u, M+1}^{r}$ is finite for $\operatorname{Re}(r)>-M / 2$ by [CPRS2], Lemma 7.2. The problem is the existence of the derivative in $u$, and for this we require a careful argument which we give in the next lemma.

We will return to the proof of Lemma 5.22 after we handle the technical issues which are summarised in the next result.

Lemma 5.23. Write $R_{u}$ for the resolvent $R_{u}=\left(\lambda-\left(s^{2}+\mathscr{D}_{u}^{2}\right)\right)^{-1}$ defined using $\mathscr{D}_{u}$. For $a_{0}, \ldots, a_{M} \in \mathcal{A}$, the product

$T_{u, j}:=\left[\mathscr{D}_{u}, a_{0}\right] R_{u}\left[\mathscr{D}_{u}, a_{1}\right] R_{u} \ldots\left[\mathscr{D}_{u}, a_{j}\right] R_{u} \mathscr{D}_{u} R_{u}\left[\mathscr{D}_{u}, a_{j+1}\right] \ldots R_{u}\left[\mathscr{D}_{u}, a_{M}\right] R_{u}$

is trace class for all $u \in[0,1]$ and is $C^{1}$ in $u$. Moreover, with $\dot{D}_{u}=-\mathscr{D}_{u} \log (|\mathscr{D}|)$ 
we obtain as expected:

$$
\begin{aligned}
& \frac{d}{d u}\left(T_{u, j}\right) \\
& =\sum_{k=0}^{M}\left[\mathscr{D}_{u}, a_{0}\right] R_{u} \ldots R_{u}\left[\mathscr{D}_{u}, a_{k}\right]\left(R_{u} 2 \mathscr{D}_{u} \dot{\mathscr{D}}_{u} R_{u}\right)\left[\mathscr{D}_{u}, a_{k+1}\right] R_{u} \ldots\left[\mathscr{D}_{u}, a_{M}\right] R_{u} \\
& +\left[\mathscr{D}_{u}, a_{0}\right] R_{u} \ldots R_{u}\left[\mathscr{D}_{u}, a_{j}\right] R_{u} D_{u}\left(R_{u} 2 \mathscr{D}_{u} \dot{\mathcal{D}}_{u} R_{u}\right)\left[\mathscr{D}_{u}, a_{j+1}\right] \ldots R_{u}\left[\mathscr{D}_{u}, a_{M}\right] R_{u} \\
& +\sum_{k=0}^{M}\left[\mathscr{D}_{u}, a_{0}\right] R_{u}\left[\mathscr{D}_{u}, a_{1}\right] R_{u} \ldots R_{u}\left[\dot{D}_{u}, a_{k}\right] R_{u} \ldots R_{u}\left[\mathscr{D}_{u}, a_{M}\right] R_{u} \\
& +\left[\mathscr{D}_{u}, a_{0}\right] R_{u}\left[\mathscr{D}_{u}, a_{1}\right] R_{u} \ldots R_{u}\left[\mathscr{D}_{u}, a_{j}\right] R_{u} \dot{\mathscr{D}}_{u} R_{u}\left[\mathscr{D}_{u}, a_{j+1}\right] \ldots R_{u}\left[\mathscr{D}_{u}, a_{M}\right] R_{u} .
\end{aligned}
$$

Proof. First we employ the estimate for $a^{*}=-a$, which is equation 10.58 in [GVF],

$$
-\|[\mathscr{D}, a]\||\mathscr{D}|^{-u} \leq\left[\mathscr{D}_{u}, a\right] \leq\|[\mathscr{D}, a]\||\mathscr{D}|^{-u}
$$

to deduce that $\left[\mathscr{D}_{u}, a\right] \in \mathscr{L}^{q^{+} / u}$ where we write $q^{+}$to indicate $q+\varepsilon$ for all positive $\varepsilon$. Using $\mathscr{D}_{u} \in O P^{1-u}$, we deduce that $R_{u} \in O P^{-2+2 u}$ and so $R_{u} \in \mathscr{L}^{q^{+} / 2(1-u)}$. The operator $T_{u, j}$ has $M+2$ factors of $R_{u}$, one factor of $\mathscr{D}_{u}$ and $M+1$ factors $\left[\mathscr{D}_{u}, a_{j}\right]$, and so $T \in \mathscr{L}^{r}$ where

$$
\frac{1}{r}=(M+1) \frac{u}{q^{+}}+(M+3 / 2) \frac{2(1-u)}{q^{+}}=\frac{2 M+3-(M+2) u}{q^{+}}
$$

The worst possible case is when $u=1$ and we find that $1 / r \geq(M+1) / q^{+}>1$, and so $T \in \mathscr{L}^{1}$ for all $u \in[0,1]$. The strict inequality follows since $M=2 N-P$ and $q-1<2 N-P \leq q+1$.

The strict inequality also implies the following. There exists $\rho>0$ such that for all $u \in[0,1], T_{u, j} \mid D^{\rho} \in \mathscr{L}^{1}$. We fix some such choice of $\rho$ from now on. Now $\left[\mathscr{D}_{u}, a_{j}\right]|\mathcal{D}|^{u}$ is order zero for all $u$ and uniformly $\leq\left\|\left[\mathscr{D}, a_{j}\right]\right\|$, and $R_{u}|\mathcal{D}|^{-2 u}$ is of order -2 uniformly in $u$. We want to write $T_{u, j}$ as a product of terms of order 0 and -2 (and a single term of order 1). To do this, we insert in the expression for $T_{u, j}$ a factor of $|\mathcal{D}|^{-2 u}$ next to each individual $R_{u}$ and a factor of $|\mathscr{D}|^{u}$ to the right of each commutator $[\mathscr{D}, a]$ : we are then forced to insert a factor of $|\mathcal{D}|^{u}$ to the left of the next commutator. In order to make the factors near the second commutator have total order 0 we are forced into adding a factor of $|\mathscr{D}|^{-u}$ to the right of the second commutator which in turn forces us to insert another factor of $|\mathcal{D}|^{u}$, etc. We make another adjustment when we get to the term $R_{u} \mathscr{D}_{u} R_{u}=R_{u} \mathscr{D}|\mathscr{D}|^{-u} R_{u}$, which force 
further adjustments after that term. Thus we obtain:

$$
\begin{aligned}
T_{u, j}= & {\left[\mathscr{D}_{u}, a_{0}\right]|\mathscr{D}|^{u} R_{u}|\mathscr{D}|^{-2 u}|\mathscr{D}|^{u}\left[\mathscr{D}_{u}, a_{1}\right]|\mathscr{D}|^{u}|\mathscr{D}|^{-u} R_{u}|\mathscr{D}|^{-2 u} } \\
& \cdot|\mathscr{D}|^{2 u}\left[\mathscr{D}_{u}, a_{2}\right]|\mathscr{D}|^{-u} \ldots|\mathscr{D}|^{j u}\left[\mathscr{D}_{u}, a_{j}\right]|\mathscr{D}|^{-(j-1) u} R_{u}|\mathscr{D}|^{-2 u} \mathscr{D} \\
& \cdot R_{u}|\mathscr{D}|^{-2 u}|\mathscr{D}|^{(j+2) u}\left[\mathscr{D}_{u}, a_{j+1}\right]|\mathscr{D}|^{-(j+1) u} \ldots \\
& \ldots|\mathscr{D}|^{(M+1) u}\left[\mathscr{D}_{u}, a_{M}\right]|\mathscr{D}|^{-M u} R_{u}|\mathscr{D}|^{-2 u}|\mathscr{D}|^{(M+2) u} \\
= & {\left[\mathscr{D}_{u}, a_{0}\right]|\mathscr{D}|^{u} R_{u}|\mathscr{D}|^{-2 u} R_{0}^{-1} R_{0}|\mathscr{D}|^{u}\left[\mathscr{D}_{u}, a_{1}\right] R_{u}|\mathscr{D}|^{-2 u} R_{0}^{-1} R_{0} } \\
& \cdot|\mathscr{D}|^{2 u}\left[\mathscr{D}_{u}, a_{2}\right]|\mathscr{D}|^{-u} \ldots|\mathscr{D}|^{j u}\left[\mathscr{D}_{u}, a_{j}\right]|\mathscr{D}|^{-(j-1) u} R_{u}|\mathscr{D}|^{-2 u} R_{0}^{-1} R_{0} \mathscr{D} \\
& \cdot R_{u}|\mathscr{D}|^{-2 u} R_{0}^{-1} R_{0}|\mathscr{D}|^{(j+2) u}\left[\mathscr{D}_{u}, a_{j+1}\right]|\mathscr{D}|^{-(j+1) u} \ldots \\
& \ldots|\mathscr{D}|^{(M+1) u}\left[\mathscr{D}_{u}, a_{m}\right]|\mathscr{D}|^{-M u}|\mathscr{D}|^{-2 u} R_{u} R_{0}^{-1} R_{0}|\mathscr{D}|^{(M+2) u} .
\end{aligned}
$$

In the first equality we have expressed $T_{u, j}$ as a product of operators of order zero (the operators $|\mathscr{D}|^{k u}\left[\mathscr{D}_{u}, a_{k}\right]|\mathscr{D}|^{-(k-1) u}$ and $|\mathscr{D}|^{(i-1) u}\left[\mathscr{D}_{u}, a_{i}\right]|\mathscr{D}|^{-i u}$ are of order zero by the pseudo-differential calculus), operators of order $-2,\left(R_{u}|\mathscr{D}|^{-2 u}\right)$ and a final term $|\mathscr{D}|^{(M+2) u}$ of order $(M+2) u \leq M+2$.

In the second equality we observe that (assuming $\lambda=a+i v$ where $a=$ $\delta / 2$ ) there is a uniform estimate which is a consequence of the functional calculus:

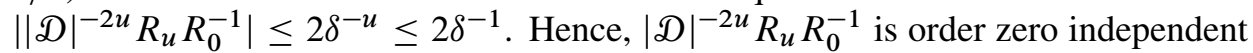
of the values of $u$, and so

$$
T_{u, j}=A_{0} R_{0} A_{1} R_{0} \ldots A_{j} R_{0} D R_{0} \ldots A_{m} R_{0}|D|^{(M+2) u},
$$

where the order zero operators $A_{j}$ are now $u$-dependent.

Obviously

$$
T_{u, j}=T_{u, j}|D|^{M+1-\rho}|\mathscr{D}|^{-M-1+\rho},
$$

but by our choice of $\rho,|D|^{-M-1+\rho} \in \mathscr{L}^{1}$, and $T_{u, j}|\mathscr{D}|^{M+1-\rho}$ is uniformly bounded in $\lambda, s, u$ and, uniformly in these parameters, of order $-\rho$ as a pseudo-differential operator.

We now want to consider the difference quotients,

$$
\frac{1}{\varepsilon}\left(T_{u+\varepsilon, j}|\mathscr{D}|^{M+1-\rho}-T_{u, j}|\mathscr{D}|^{M+1-\rho}\right) .
$$

Observe that these differences lie in $O P^{-\rho}$ and so are bounded. In order to take the limit as $\varepsilon \rightarrow 0$, we require some elementary estimates. If $t$ lies between $x$ and 1 , we have

$$
\int_{1}^{t} s^{-1-\varepsilon} d s \leq \int_{1}^{x} s^{-1-\varepsilon} d s=\frac{1}{\varepsilon}\left(1-x^{-\varepsilon}\right) .
$$


So let $x \in[\sqrt{\delta}, \infty)$, where $\mathscr{D}^{2} \geq \delta$, then we have the estimate

$$
\begin{aligned}
\int_{1}^{x}\left(t^{-1}-t^{-1-\varepsilon}\right) d t & =-\int_{1}^{x} t^{-1}\left(t^{-\varepsilon}-1\right) d t \\
& =-\int_{1}^{x} t^{-1}\left(-\varepsilon \int_{1}^{t} s^{-1-\varepsilon} d s\right) d t \\
& \leq \varepsilon \int_{1}^{x} t^{-1} \int_{1}^{x} s^{-1-\varepsilon} d s d t=\left(1-x^{-\varepsilon}\right) \log x
\end{aligned}
$$

Hence

$$
\left|\frac{1}{\varepsilon}\left(x^{-\varepsilon}-1\right)+\log x\right| \leq\left(1-x^{-\varepsilon}\right) \log x .
$$

Since $|\log x| \leq C x^{\rho}$ for all $x \geq \delta$, for any $\rho>0$, the functional calculus then gives us the inequality

$$
\left\|\left(\frac{1}{\varepsilon}\left(|\mathscr{D}|^{-\varepsilon}-1\right)+\log |\mathscr{D}|\right)|\mathscr{D}|^{-\rho}\right\| \leq C\left\|1-|\mathscr{D}|^{-\varepsilon}\right\|\left\||\mathscr{D}|^{-\rho} \log |\mathscr{D}|\right\| \rightarrow 0 \text { as } \varepsilon \rightarrow 0 \text {. }
$$

Thus

$$
\lim _{\varepsilon \rightarrow 0} \frac{1}{\varepsilon}\left(|\mathscr{D}|^{-\varepsilon}-1\right)|\mathscr{D}|^{-\rho}=-|\mathscr{D}|^{-\rho} \log |\mathscr{D}|
$$

where the limit is taken in the norm topology. Since we are using the norm topology and both sides lie in $O P^{0}$, we may regard this as an equality in $O P^{0}$.

We will now show that the limit

$$
\lim _{\varepsilon \rightarrow 0} \frac{1}{\varepsilon}\left(T_{(u+\varepsilon), j}-T_{u, j}\right)|\mathcal{D}|^{M+1-\rho}
$$

also exists in $O P^{0}$ in the norm topology, and therefore $\lim _{\varepsilon \rightarrow 0} \frac{1}{\varepsilon}\left(T_{(u+\varepsilon), j}-T_{u, j}\right)$ exists in the $\mathscr{L}^{1}$ norm. Our earlier computations show that we can write

$$
T_{u, j}=B_{0}(u) \mathcal{R}_{u} B_{1}(u) \mathcal{R}_{u} \ldots B_{j}(u) \mathcal{R}_{u} \mathcal{D} \mathcal{R}_{u} \ldots \mathcal{R}_{u} B_{M}(u) \mathcal{R}_{u},
$$

where $\mathcal{R}_{u}=R_{u}|\mathcal{D}|^{-2 u} \in O P^{-2}$ and, for each $k=0, \ldots, M$,

$$
B_{k}(u)=|\mathscr{D}|^{k u}\left[\mathscr{D}_{u}, a_{k}\right]|\mathscr{D}|^{-(k-1) u} \quad \text { or } \quad B_{k}(u)=|\mathscr{D}|^{(k-1) u}\left[\mathscr{D}_{u}, a_{k}\right]|\mathscr{D}|^{-k u} \text {, }
$$

depending on whether $k \leq j$ or $k>j$, and in each case $B_{k}(u) \in O P^{0}$. So now we can write

$$
\begin{aligned}
& \frac{1}{\varepsilon}\left(T_{u+\varepsilon}-T_{u}\right)|\mathcal{D}|^{M+1-\rho} \\
& =\frac{1}{\varepsilon}\left(B_{0}(u+\varepsilon)-B_{0}(u)\right)|\mathscr{D}|^{-\rho}|\mathcal{D}|^{\rho} \mathcal{R}_{u+\varepsilon} B_{1}(u+\varepsilon) \ldots B_{M}(u+\varepsilon) \mathcal{R}_{u+\varepsilon}|\mathcal{D}|^{M+1-\rho} \\
& +\frac{1}{\varepsilon} B_{0}(u)\left(\mathcal{R}_{u+\varepsilon}-\mathcal{R}_{u}\right)|\mathcal{D}|^{-\rho}|\mathcal{D}|^{\rho} B_{1}(u+\varepsilon) \mathcal{R}_{u+\varepsilon} \ldots B_{M}(u+\varepsilon) \mathcal{R}_{u+\varepsilon}|\mathcal{D}|^{M+1-\rho} \\
& +\cdots+\frac{1}{\varepsilon} B_{0}(u) \mathcal{R}_{u} B_{1}(u) \ldots B_{M}(u)\left(\mathcal{R}_{u+\varepsilon}-\mathcal{R}_{u}\right)|\mathcal{D}|^{-\rho}|\mathcal{D}|^{\rho}|\mathcal{D}|^{M+1-\rho}
\end{aligned}
$$


We have written each term as a product $\alpha \beta \gamma$, where the sum of orders of $\alpha$ and $\gamma$ is two or zero, while $\beta$ is a difference quotient times $|\mathcal{D}|^{-\rho}$, and so the order of $\beta$ is always either $-2-\rho$ or $-\rho$. Below we show that the two possibilities for $\beta$ give norm convergent limits, in $O P^{-2}$ or $O P^{0}$, which will imply that $T_{u, j}$ is differentiable, by a standard argument. The continuity of the resulting derivative can be determined by similar, but simpler, arguments.

So we now examine the difference quotients. There are two kinds of terms which arise in this computation (the factor of $|\mathcal{D}|^{-u}$ arising from the extra $\mathscr{D}_{u}$ is included in our other factors). First we consider $\mathcal{R}_{u+\varepsilon}-\mathcal{R}_{u}$ which equals

$$
\begin{aligned}
R_{u+\varepsilon} & |\mathscr{D}|^{-2(u+\varepsilon)}-R_{u}|\mathscr{D}|^{-2 u} \\
& =R_{u+\varepsilon}|\mathscr{D}|^{-2(u+\varepsilon)}-R_{u}|\mathscr{D}|^{-2(u+\varepsilon)}+R_{u}|\mathscr{D}|^{-2(u+\varepsilon)}-R_{u}|\mathscr{D}|^{-2 u} \\
& =R_{u+\varepsilon}|\mathscr{D}|^{-2(u+\varepsilon)}\left(|\mathscr{D}|^{-2 \varepsilon}-1\right) \mathscr{D}_{u}^{2} R_{u}+R_{u}|\mathscr{D}|^{-2 u}\left(|\mathscr{D}|^{-2 \varepsilon}-1\right) .
\end{aligned}
$$

Observe we have chosen to add and subtract a term which lies in $O P^{-2}$. Since the factor $R_{u}|\mathscr{D}|^{-2 u}=R_{u}|\mathscr{D}|^{-2 u} R_{0}^{-1} R_{0}$ is uniformly in $O P^{-2}$, the same method of proof as before shows that

$\lim _{\varepsilon \rightarrow 0} \frac{1}{\varepsilon}\left(R_{u+\varepsilon}|\mathscr{D}|^{-2(u+\varepsilon)}-R_{u}|\mathscr{D}|^{-2 u}\right)|\mathscr{D}|^{-\rho}=R_{u}|\mathscr{D}|^{-2 u} 2 \log |\mathscr{D}|\left(\mathscr{D}_{u}^{2} R_{u}-1\right)|\mathcal{D}|^{-\rho}$

where the limit exists in norm and lies in $O P^{-2}$.

The second kind of difference quotient is (one of the two forms of) $B_{n}(u+\varepsilon)-$ $B_{n}(u)$, and we will present the proof for the case

$$
B_{n}(u)=|\mathscr{D}|^{n u}\left[\mathscr{D}_{u}, a_{n}\right]|\mathscr{D}|^{-(n-1) u},
$$

the other possibility being entirely similar. By adding and subtracting terms of order strictly less than zero, we can rewrite this difference as

$$
\begin{aligned}
& |\mathscr{D}|^{n(u+\varepsilon)}\left[\mathscr{D}_{u+\varepsilon}, a\right]|\mathscr{D}|^{-(n-1)(u+\varepsilon)}-|\mathscr{D}|^{n u}\left[\mathscr{D}_{u}, a\right]|\mathscr{D}|^{-(n-1) u} \\
& =|\mathscr{D}|^{n(u+\varepsilon)}\left[\mathscr{D}_{u+\varepsilon}, a\right]|\mathscr{D}|^{-(n-1)(u+\varepsilon)}-|\mathscr{D}|^{n(u+\varepsilon)}\left[\mathscr{D}_{u+\varepsilon}, a\right]|\mathscr{D}|^{-(n-1) u} \\
& +|\mathscr{D}|^{n(u+\varepsilon)}\left[\mathscr{D}_{u+\varepsilon}, a\right]|\mathscr{D}|^{-(n-1) u}-|\mathscr{D}|^{n u}\left[\mathscr{D}_{u+\varepsilon}, a\right]|\mathscr{D}|^{-(n-1) u} \\
& +|\mathcal{D}|^{n u}\left[\mathscr{D}_{u+\varepsilon}, a\right]|\mathscr{D}|^{-(n-1) u}-|\mathscr{D}|^{n u}\left[\mathscr{D}_{u}, a\right]|\mathscr{D}|^{-(n-1) u} \\
& =|\mathscr{D}|^{n(u+\varepsilon)}\left[\mathscr{D}_{u+\varepsilon}, a\right]|\mathscr{D}|^{-(n-1) u}\left(|\mathscr{D}|^{-(n-1) \varepsilon}-1\right)+\left(1-|\mathscr{D}|^{-n \varepsilon}\right)|\mathscr{D}|^{n(u+\varepsilon)} \\
& \cdot\left[\mathscr{D}_{u+\varepsilon}, a\right]|\mathscr{D}|^{-(n-1) u}+|\mathscr{D}|^{n u}\left[\mathscr{D}_{u+\varepsilon}-\mathscr{D}_{u}, a\right]|\mathscr{D}|^{-(n-1) u} \text {. }
\end{aligned}
$$

The first term can be handled using our estimates above, the second term can be handled similarly by using the factor of $|\mathscr{D}|^{\rho}|\mathcal{D}|^{-\rho}$ on the left so only the last term needs examination. We rewrite this last term as

$$
\begin{aligned}
& |\mathscr{D}|^{n u}\left[\mathscr{D}_{u+\varepsilon}-\mathscr{D}_{u}, a\right]|\mathscr{D}|^{-(n-1) u} \\
& \quad=|\mathscr{D}|^{n u} \mathscr{D}\left[|\mathscr{D}|^{-(u+\varepsilon)}-|\mathscr{D}|^{-u}, a\right]|\mathscr{D}|^{-(n-1) u}+|\mathscr{D}|^{n u}[\mathscr{D}, a]|\mathscr{D}|^{-n u}\left(|\mathscr{D}|^{-\varepsilon}-1\right) .
\end{aligned}
$$


The second of these terms can also be dealt with using our previous methods, and so we are left with examining

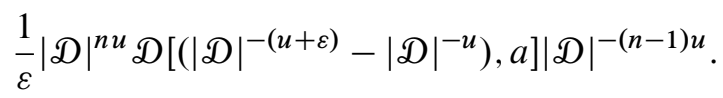

We proceed in stages. Recalling that this difference quotient is multiplied by $|\mathscr{D}|^{-\rho}$, we consider the convergence, in $O P^{-\rho} \subset O P^{0}$, of

$$
\frac{1}{\varepsilon}|\mathscr{D}|^{n u} \mathscr{D}\left[\left(|\mathscr{D}|^{-(u+\varepsilon)}-|\mathscr{D}|^{-u}\right), a\right]|\mathscr{D}|^{-\rho}|\mathscr{D}|^{-(n-1) u} .
$$

Applying the Leibnitz rule we have

$$
\begin{array}{rl}
\frac{1}{\varepsilon}|\mathscr{D}|^{n u} & \mathscr{D}\left[\left(|\mathscr{D}|^{-(u+\varepsilon)}-|\mathscr{D}|^{-u}\right), a\right]|\mathscr{D}|^{-\rho}|\mathscr{D}|^{-(n-1) u} \\
= & \frac{1}{\varepsilon}|\mathscr{D}|^{(n-1) u} \mathscr{D}\left[\left(|\mathscr{D}|^{-\varepsilon}-1\right), a\right]|\mathscr{D}|^{-\rho}|\mathscr{D}|^{-(n-1) u} \\
& +\frac{1}{\varepsilon}|\mathscr{D}|^{n u} \mathscr{D}\left[|\mathscr{D}|^{-u}, a\right]\left(|\mathscr{D}|^{-\varepsilon}-1\right)|\mathscr{D}|^{-\rho}|\mathscr{D}|^{-(n-1) u} .
\end{array}
$$

As $|D|^{u} \mathscr{D}\left[|D|^{-u}, a\right] \in O P^{0}$ and conjugation by $|\mathscr{D}|^{(n-1) u}$ preserves $O P^{0}$, we see using our previous methods that the second term has a limit in $O P^{0}$. For the first term we employ the Leibnitz rule again. This gives us

$$
\begin{aligned}
\frac{1}{\varepsilon}|\mathscr{D}|^{(n-1) u} \mathscr{D}\left[\left(|\mathscr{D}|^{-\varepsilon}-1\right), a\right]|\mathscr{D}|^{-\rho}|\mathscr{D}|^{-(n-1) u} \\
=\frac{1}{\varepsilon}|\mathscr{D}|^{(n-1) u} \mathscr{D}\left[\left(|\mathscr{D}|^{-\varepsilon}-1\right)|\mathscr{D}|^{-\rho}, a\right]|\mathscr{D}|^{-(n-1) u} \\
\quad+\frac{1}{\varepsilon}|\mathscr{D}|^{(n-1) u}\left(|\mathscr{D}|^{-\varepsilon}-1\right)|\mathscr{D}|^{-\rho} \mathscr{D}\left[|\mathscr{D}|^{\rho}, a\right]|\mathscr{D}|^{-\rho}|\mathscr{D}|^{-(n-1) u} .
\end{aligned}
$$

As before conjugation by $|\mathscr{D}|^{(n-1) u}$ does not affect matters. For the first term we observe that $\mathscr{D}\left[\frac{1}{\varepsilon}\left(|D|^{-\varepsilon}-1\right)|D|^{-\rho}, a\right]$ is uniformly in $O P^{0}$ and has a limit as $\varepsilon \rightarrow 0$ by our previous methods. The proof is completed by noting that the second term is handled similarly once we see that

$$
\mathscr{D}\left[|\mathscr{D}|^{\rho}, a\right]|\mathscr{D}|^{-\rho}=-\mathscr{D}|\mathscr{D}|^{\rho}\left[|\mathscr{D}|^{-\rho}, a\right] \in O P^{0} .
$$

Proof of Lemma 5.22. Lemma 5.23, taken together with arguments of a similar nature, shows that $\Psi_{u, M}^{r}$ and $\frac{d}{d u} \Phi_{u, M+1}^{r}$ are well defined and continuous. The proof of Lemma 5.23 also shows that the formal differentiations given below are in fact justified.

First of all, using the $\mathscr{D}_{u}$ version of equation 5.5 of Proposition 5.14 and the $R_{u}$ version of Definition 5.4 to expand $\left(B \Phi_{u, M+1}^{r}\right)\left(a_{0}, \ldots, a_{M}\right)$, we see that it is the sum 
of the $T_{u, j}$ and so its derivative is the sum over $j$ of the derivatives in Lemma 5.23. Using the $R_{u}$ version of Definition 5.4 again to rewrite this in terms of $\langle\langle\ldots\rangle\rangle$ where possible we obtain:

$$
\begin{aligned}
& \frac{d}{d u}\left(B \Phi_{u, M+1}^{r}\right)\left(a_{0}, \ldots, a_{M}\right) \\
& =-\frac{\eta_{M}}{2} \int_{0}^{\infty} s^{M} \sum_{i=0}^{M}\left(\left\langle\left\langle\left[\mathscr{D}_{u}, a_{0}\right], \ldots,\left[\mathscr{D}_{u}, a_{i}\right], 2 \mathscr{D}_{u} \dot{\mathscr{D}}_{u}, \ldots,\left[\mathscr{D}_{u}, a_{M}\right]\right\rangle\right\rangle_{M+1, s, r}\right. \\
& \left.\quad+\left\langle\left\langle\left[\mathscr{D}_{u}, a_{0}\right], \ldots,\left[\dot{D}_{u}, a_{i}\right], \ldots,\left[\mathscr{D}_{u}, a_{M}\right]\right\rangle\right\rangle_{M, s, r}\right) d s-\frac{\eta_{M}}{2} \\
& \quad \cdot \int_{0}^{\infty} s^{M} \sum_{i=0}^{M}(-1)^{i}\left\langle\left[\mathscr{D}_{u}, a_{0}\right], \ldots,\left[\mathscr{D}_{u}, a_{i}\right], \dot{D}_{u}, \ldots,\left[\mathscr{D}_{u}, a_{M}\right]\right\rangle_{M+1, s, r} d s .
\end{aligned}
$$

For the next step we compute $B b \Psi_{u, M}^{r}$ and then use $b B=-B b$. First we apply $b$ :

$$
\begin{aligned}
& \left(b \Psi_{u, M}^{r}\right)\left(a_{0}, \ldots, a_{M+1}\right) \\
& =-\frac{\eta_{M}}{2} \sum_{j=1}^{M}(-1)^{j} \int_{0}^{\infty} s^{M}\left\langle\left\langle a_{0} \dot{\mathscr{D}}_{u}, \ldots,\left[\mathscr{D}_{u}, a_{j} a_{j+1}\right], \ldots,\left[\mathscr{D}_{u}, a_{M+1}\right]\right\rangle\right\rangle_{M, s, r} d s \\
& -\frac{\eta_{M}}{2} \int_{0}^{\infty} s^{M}\left\langle\left\langle a_{0} a_{1} \dot{\mathscr{D}}_{u},\left[\mathscr{D}_{u}, a_{2}\right], \ldots,\left[\mathscr{D}_{u}, a_{M+1}\right]\right\rangle\right\rangle_{M, s, r} d s \\
& -(-1)^{M+1} \frac{\eta_{M}}{2} \int_{0}^{\infty} s^{M}\left\langle\left\langle a_{M+1} a_{0} \dot{\mathscr{D}}_{u},\left[\mathscr{D}_{u}, a_{1}\right], \ldots,\left[\mathscr{D}_{u}, a_{M}\right]\right\rangle\right\rangle_{M, s, r} d s \\
& =-\frac{\eta_{M}}{2} \sum_{j=1}^{M}(-1)^{j} \int_{0}^{\infty} s^{M}\left\langle\left\langle a_{0} \dot{\mathscr{D}}_{u}, \ldots, a_{j}\left[\mathscr{D}_{u}, a_{j+1}\right]\right.\right. \\
& \left.\left.+\left[\mathscr{D}_{u}, a_{j}\right] a_{j+1}, \ldots,\left[\mathscr{D}_{u}, a_{M+1}\right]\right\rangle\right\rangle_{M, s, r} d s \\
& -\frac{\eta_{M}}{2} \int_{0}^{\infty} s^{M}\left\langle\left\langle a_{0} \dot{D}_{u} a_{1},\left[\mathscr{D}_{u}, a_{2}\right], \ldots,\left[\mathscr{D}_{u}, a_{M+1}\right]\right\rangle\right\rangle_{M, s, r} d s \\
& +\frac{\eta_{M}}{2} \int_{0}^{\infty} s^{M}\left\langle\left\langle a_{0}\left[\dot{\mathscr{D}}_{u}, a_{1}\right],\left[\mathscr{D}_{u}, a_{2}\right], \ldots,\left[\mathscr{D}_{u}, a_{M+1}\right]\right\rangle\right\rangle_{M, s, r} d s \\
& -(-1)^{M+1} \frac{\eta_{M}}{2} \int_{0}^{\infty} s^{M}\left\langle\left\langle a_{M+1} a_{0} \dot{D}_{u},\left[\mathscr{D}_{u}, a_{1}\right], \ldots,\left[\mathscr{D}_{u}, a_{M}\right]\right\rangle\right\rangle_{M, s, r} d s \\
& =-\frac{\eta_{M}}{2} \int_{0}^{\infty} s^{M} \sum_{j=1}^{M+1}(-1)^{j}\left\langle\left\langle a_{0} \dot{\mathscr{D}}_{u},\left[\mathscr{D}_{u}, a_{1}\right], \ldots,\left[\mathscr{D}_{u}^{2}, a_{j}\right], \ldots\right.\right. \\
& \left.\left.\ldots,\left[\mathscr{D}_{u}, a_{M+1}\right]\right\rangle\right\rangle_{M+1, s, r} d s
\end{aligned}
$$




$$
\begin{aligned}
& -\frac{\eta_{M}}{2} \int_{0}^{\infty} s^{M} \sum_{j=1}^{M+1}(-1)^{j}(-1)^{\operatorname{deg}\left(a_{0} \dot{\mathscr{D}}_{u}\right)+\cdots+\operatorname{deg}\left(\left[\mathscr{D}_{u}, a_{j-1}\right]\right)} \\
& \left\langle a_{0} \dot{\mathscr{D}}_{u},\left[\mathscr{D}_{u}, a_{1}\right], \ldots,\left[\mathscr{D}_{u}, a_{M+1}\right]\right\rangle_{M+1, s, r} d s \\
& +\frac{\eta_{M}}{2} \int_{0}^{\infty} s^{M}\left\langle\left\langle a_{0}\left[\dot{\mathscr{D}}_{u}, a_{1}\right], \ldots,\left[\mathscr{D}_{u}, a_{M+1}\right]\right\rangle_{M, s, r} d s .\right.
\end{aligned}
$$

The last equality follows from the $R_{u}$ version of Lemma 5.5. In the above we note that $\operatorname{deg}\left(a_{0} \dot{\mathscr{D}}_{u}\right)=1=\operatorname{deg}\left(\left[\mathscr{D}_{u}, a_{k}\right]\right)$ for all $k$ so that $\operatorname{deg}\left(a_{0} \dot{\mathscr{D}}_{u}\right)+\cdots$ $\cdots+\operatorname{deg}\left(\left[\mathscr{D}_{u}, a_{j-1}\right]\right)=j$ and $\operatorname{deg}\left(a_{0} \dot{D}_{u}\right)+\cdots+\operatorname{deg}\left(\left[\mathscr{D}_{u}, a_{M+1}\right]\right)=M+2 \equiv$ $P(\bmod 2)$. We also note the commutator identity $\left[\mathscr{D}_{u}^{2}, a_{j}\right]=\left\{\mathscr{D}_{u},\left[\mathscr{D}_{u}, a_{j}\right]\right\}=$ $\left[\mathscr{D}_{u},\left[\mathscr{D}_{u}, a_{j}\right]\right]_{ \pm}$. So in order to apply the $\mathscr{D}_{u}$ version of equation (5.3) of Lemma 5.5 we first add and subtract

$$
-\frac{\eta_{M}}{2} \int_{0}^{\infty} s^{M}\left\langle\left\langle\left\{\mathscr{D}_{u}, a_{0} \dot{\mathscr{D}}_{u}\right\},\left[\mathscr{D}_{u}, a_{1}\right], \ldots,\left[\mathscr{D}_{u}, a_{M+1}\right]\right\rangle\right\rangle_{M+1, s, r} d s
$$

and then apply equation (5.4) to obtain:

$$
\begin{aligned}
& \left(b \Psi_{u, M}^{r}\right)\left(a_{0}, \ldots, a_{M+1}\right) \\
& =-2 \frac{\eta_{M}}{2} \int_{0}^{\infty} s^{M} \sum_{j=0}^{M+1}\left\langle a_{0} \dot{\mathscr{D}}_{u}, \ldots,\left[\mathscr{D}_{u}, a_{j}\right], \mathscr{D}_{u}^{2}, \ldots,\left[\mathscr{D}_{u}, a_{M+1}\right]\right\rangle_{M+2, s, r} d s \\
& \quad+\frac{\eta_{M}}{2} \int_{0}^{\infty} s^{M}\left\langle\left\langle a_{0}\left\{\mathscr{D}_{u}, \dot{\mathscr{D}}_{u}\right\}+\left[\mathscr{D}_{u}, a_{0}\right] \dot{\mathscr{D}}_{u},\left[\mathscr{D}_{u}, a_{1}\right], \ldots,\left[\mathscr{D}_{u}, a_{M+1}\right]\right\rangle\right\rangle_{M+1, s, r} d s \\
& \quad-\frac{\eta_{M}}{2}(M+1) \int_{0}^{\infty} s^{M}\left\langle a_{0} \dot{\mathscr{D}}_{u},\left[\mathscr{D}_{u}, a_{1}\right], \ldots,\left[\mathscr{D}_{u}, a_{M+1}\right]\right\rangle_{M+1, s, r} d s \\
& \quad+\frac{\eta_{M}}{2} \int_{0}^{\infty} s^{M}\left\langle\left\langle a_{0}\left[\dot{D}_{u}, a_{1}\right], \ldots,\left[\mathscr{D}_{u}, a_{M+1}\right]\right\rangle\right\rangle_{M, s, r} d s .
\end{aligned}
$$

Then we apply the $\mathscr{D}_{u}$ version of Lemma 5.8 as modified in the proof of Proposition 5.14 with $t=0$ to the first term above to obtain

$$
\begin{aligned}
& =\frac{\eta_{M}}{2}(q+2 r) \int_{0}^{\infty} s^{M}\left\langle a_{0} \dot{\mathscr{D}}_{u},\left[\mathscr{D}_{u}, a_{1}\right], \ldots,\left[\mathscr{D}_{u}, a_{M+1}\right]\right\rangle_{M+1, s, r} d s \\
& +\frac{\eta_{M}}{2} \int_{0}^{\infty} s^{M}\left\langle\left\langle a_{0}\left\{\mathscr{D}_{u}, \dot{D}_{u}\right\}+\left[\mathscr{D}_{u}, a_{0}\right] \dot{D}_{u},\left[\mathscr{D}_{u}, a_{1}\right], \ldots,\left[\mathscr{D}_{u}, a_{M+1}\right]\right\rangle\right\rangle_{M+1, s, r} d s \\
& +\frac{\eta_{M}}{2} \int_{0}^{\infty} s^{M}\left\langle\left\langle a_{0}\left[\dot{D}_{u}, a_{1}\right], \ldots,\left[\mathscr{D}_{u}, a_{M+1}\right]\right\rangle\right\rangle_{M, s, r} d s .
\end{aligned}
$$


The next step is to apply $B$ to these three terms:

$$
\begin{aligned}
& \left(B b \Psi_{u, M}^{r}\right)\left(a_{0}, \ldots, a_{M}\right) \\
& =(q+2 r) \frac{\eta_{M}}{2} \sum_{j=0}^{M}(-1)^{(M+1) j} \int_{0}^{\infty} s^{M}\left\langle\dot{D}_{u},\left[\mathscr{D}_{u}, a_{j}\right], \ldots,\left[\mathscr{D}_{u}, a_{j-1}\right]\right\rangle_{M+1, s, r} d s \\
& +\frac{\eta_{M}}{2} \sum_{j=0}^{M}(-1)^{(M+1) j} \int_{0}^{\infty} s^{M}\left\langle\left\langle\left\{\mathscr{D}_{u}, \dot{D}_{u}\right\},\left[\mathscr{D}_{u}, a_{j}\right], \ldots,\left[\mathscr{D}_{u}, a_{j-1}\right]\right\rangle\right\rangle_{M+1, s, r} d s \\
& +\frac{\eta_{M}}{2} \sum_{j=0}^{M}(-1)^{(M+1) j} \int_{0}^{\infty} s^{M}\left\langle\left\langle\left[\dot{\mathscr{D}}_{u}, a_{j}\right], \ldots,\left[\mathscr{D}_{u}, a_{j-1}\right]\right\rangle\right\rangle_{M, s, r} d s \\
& =\frac{(q+2 r) \eta_{M}}{2} \sum_{j=0}^{M}(-1)^{(M+1) j+A j} \int_{0}^{\infty} s^{M}\left\langle\left[\mathscr{D}_{u}, a_{0}\right], \ldots,\left[\mathscr{D}_{u}, a_{j-1}\right], \dot{D}_{u}, \ldots\right. \\
& \left.\ldots,\left[\mathscr{D}_{u}, a_{M}\right]\right\rangle_{M+1, s, r} d s \\
& +\frac{\eta_{M}}{2} \sum_{j=0}^{M}(-1)^{(M+1) j+(1+A) j} \int_{0}^{\infty} s^{M}\left\langle\left\langle\left[\mathscr{D}_{u}, a_{0}\right], \ldots,\left\{\mathscr{D}_{u}, \dot{D}_{u}\right\},\left[\mathscr{D}_{u}, a_{j}\right], \ldots\right.\right. \\
& \left.\left.\ldots,\left[\mathscr{D}_{u}, a_{M}\right]\right\rangle\right\rangle_{M+1, s, r} d s \\
& +\frac{\eta_{M}}{2} \sum_{j=0}^{M}(-1)^{(M+1) j+(1+A) j} \int_{0}^{\infty} s^{M}\left\langle\left\langle\left[\mathscr{D}_{u}, a_{0}\right], \ldots,\left[\mathscr{D}_{u}, a_{j-1}\right],\left[\dot{\mathscr{D}}_{u}, a_{j}\right], \ldots\right.\right. \\
& \left.\ldots,\left[\mathscr{D}_{u}, a_{M}\right]\right\rangle_{M, s, r} d s \\
& =(q+2 r) \frac{\eta_{M}}{2} \sum_{j=0}^{M}(-1)^{j} \int_{0}^{\infty} s^{M}\left\langle\left[\mathscr{D}_{u}, a_{0}\right], \ldots,\left[\mathscr{D}_{u}, a_{j-1}\right], \dot{\mathscr{D}}_{u}, \ldots\right. \\
& \left.\ldots,\left[\mathscr{D}_{u}, a_{M}\right]\right\rangle_{M+1, s, r} d s \\
& +\frac{\eta_{M}}{2} \sum_{j=0}^{M} \int_{0}^{\infty} s^{M}\left\langle\left\langle\left[\mathscr{D}_{u}, a_{0}\right], \ldots, 2 \mathscr{D}_{u} \dot{D}_{u},\left[\mathscr{D}_{u}, a_{j}\right], \ldots,\left[\mathscr{D}_{u}, a_{M}\right]\right\rangle\right\rangle_{M+1, s, r} d s \\
& +\frac{\eta_{M}}{2} \sum_{j=0}^{M} \int_{0}^{\infty} s^{M}\left\langle\left\langle\left[\mathscr{D}_{u}, a_{0}\right], \ldots,\left[\mathscr{D}_{u}, a_{j-1}\right],\left[\dot{\mathscr{D}}_{u}, a_{j}\right], \ldots,\left[\mathscr{D}_{u}, a_{M}\right]\right\rangle\right\rangle_{M, s, r} d s .
\end{aligned}
$$

Using $b B=-B b$ and our formula for $\frac{d}{d u}\left(B \Phi_{u, M+1}^{r}\right)\left(a_{0}, \ldots, a_{M}\right)$ we obtain

$$
\begin{aligned}
& \left(b B \Psi_{u, M}^{r}\right)\left(a_{0}, \ldots, a_{M}\right) \\
& \quad=-(q+2 r) \frac{\eta_{M}}{2} \sum_{j=0}^{M}(-1)^{j} \int_{0}^{\infty} s^{M}\left\langle\left[\mathscr{D}_{u}, a_{0}\right], \ldots,\left[\mathscr{D}_{u}, a_{j-1}\right], \dot{D}_{u}, \ldots\right.
\end{aligned}
$$




$$
\begin{gathered}
\left.\ldots,\left[\mathscr{D}_{u}, a_{M}\right]\right\rangle_{M+1, s, r} d s \\
+\frac{\eta_{M}}{2} \sum_{i=0}^{M}(-1)^{i} \int_{0}^{\infty} s^{M}\left\langle\left[\mathscr{D}_{u}, a_{0}\right], \ldots,\left[\mathscr{D}_{u}, a_{i}\right], \dot{D}_{u}, \ldots\right. \\
\left.\ldots,\left[\mathscr{D}_{u}, a_{M}\right]\right\rangle_{M+1, s, r} d s+\frac{d}{d u}\left(B \Phi_{u, M+1}^{r}\right)\left(a_{0}, \ldots, a_{M}\right) .
\end{gathered}
$$

This proves the result.

Corollary 5.24. For D invertible we have

$$
\begin{aligned}
& \frac{1}{(r+(q-1) / 2)}\left(b B \Psi_{u, M}^{r}\right)\left(a_{0}, \ldots, a_{M}\right) \\
& \quad=\frac{1}{(r+(q-1) / 2)} \frac{d}{d u}\left(B \Phi_{u, M+1}^{r}\right)\left(a_{0}, \ldots, a_{M}\right)+h(r)
\end{aligned}
$$

where $h(r)$ is analytic for $\operatorname{Re}(r)>-M / 2$.

Proof. This follows from [CPRS2], Lemma 7.2, applied to the function

$$
\int_{0}^{\infty} s^{M}\left\langle\left[\mathscr{D}_{u}, a_{0}\right], \ldots,\left[\mathscr{D}_{u}, a_{i}\right], \dot{\mathcal{D}}_{u}, \ldots,\left[\mathscr{D}_{u}, a_{M}\right]\right\rangle_{M+1, s, r} d s,
$$

which shows that this function is holomorphic for $\operatorname{Re}(r)>-M / 2$, and in both the even and odd cases, $-M / 2<(1-q) / 2$.

Corollary 5.25. For D invertible we have

$$
\left(b B \Psi_{u, M}^{(1-q) / 2}\right)\left(a_{0}, \ldots, a_{M}\right)=\frac{d}{d u}\left(B \Phi_{u, M+1}^{(1-q) / 2}\right)\left(a_{0}, \ldots, a_{M}\right) .
$$

Proof. We have already observed that $B \Phi_{u, M+1}^{r}$ is holomorphic at $r=(1-q) / 2$, and so by Corollary 5.24 we can take the residues of both sides of equation (5.7) to obtain the result. Observe that taking these residues did not require isolated spectral dimension.

\subsection{Proofs of Theorems 5.1 and 5.2}

Proof of Theorem 5.1. The image of the cyclic cochain

$$
\frac{1}{(r-(1-q) / 2)} \int_{0}^{1} B \Psi_{u, M}^{r}\left(a_{0}, \ldots, a_{M}\right) d u
$$

under the operator $b$ is given by

$$
\frac{1}{(r-(1-q) / 2)} \int_{0}^{1} \frac{d}{d u} B \Phi_{u, M+1}^{r}\left(a_{0}, \ldots, a_{M}\right) d u+\text { holo. }
$$


Here holo is the integral over $u$ of the holomorphic remainder from Corollary 5.24. Integrating this remainder in $u$ does not affect the estimates proving holomorphy at $r=(1-q) / 2$, since the integral is absolutely convergent. By the fundamental theorem of calculus, equation (5.8) is (modulo functions holomorphic at $r=(1-q) / 2$ ) the difference of $\frac{1}{(r-(1-q) / 2)} B \Phi_{M+1}^{r}$ defined using $F=\mathscr{D}|\mathscr{D}|^{-1}$ and $\frac{1}{(r-(1-q) / 2)} B \Phi_{M+1}^{r}$ defined using $\mathscr{D}$. Hence the two are cohomologous in cyclic cohomology. Recalling that $F^{2}=1$ and using our previous formula for $B \Phi_{u, m}^{r}$ (the $\mathscr{D}_{u}$ version of Proposition 5.14 with $u=1$ ) we have

$$
\begin{gathered}
\left.\left(B \Phi_{M+1}^{r}\right)\left(a_{0}, \ldots, a_{M}\right)\right|_{u=1} \\
=-\frac{\eta_{M}}{2} \sum_{j=0}^{M}(-1)^{j+1} \int_{0}^{\infty} s^{M}\left\langle\left[F, a_{0}\right], \ldots,\left[F, a_{j}\right], F,\left[F, a_{j+1}\right], \ldots\right. \\
\left.\ldots,\left[F, a_{M}\right]\right\rangle_{M+1, s, r} d s \\
=-\frac{\eta_{M}}{2} \sum_{j=0}^{M} \int_{0}^{\infty} s^{M} \frac{1}{2 \pi i} \tau\left(\gamma \int_{l} \lambda^{-q / 2-r} F\left[F, a_{0}\right] \ldots\right. \\
=\frac{\eta_{M}}{2} \frac{(-1)^{M}}{M !} \frac{\Gamma(M+1+q / 2+r)}{\Gamma(q / 2+r)} \int_{0}^{\infty} s^{M} \tau\left(\gamma F\left[F, a_{0}\right] \ldots\right. \\
\left.\ldots\left[F, a_{M}\right]\left(s^{2}+1\right)^{-M-1-q / 2-r}\right) d s .
\end{gathered}
$$

In the second equality we anticommuted $F$ past the commutators and pulled all the resolvents to the right (they commute with everything since they involve only scalars). In the last equality we used the Cauchy integral formula to do the contour integral and performed the sum.

Now we pull out $\left(s^{2}+1\right)^{-M-1-p / 2-r}$ from the trace, leaving the identity behind. The $s$ integral is as follows.

$$
\begin{aligned}
\int_{0}^{\infty} s^{M} & \left(s^{2}+1\right)^{-M-1-p / 2-r} d s \\
& =\frac{1}{\Gamma(M+1+q / 2+r)} \int_{0}^{\infty} \int_{0}^{\infty} s^{M} u^{M+q / 2+r} e^{-u\left(s^{2}+1\right)} d u d s \\
& =\frac{1}{\Gamma(M+1+q / 2+r)} \int_{0}^{\infty} \int_{0}^{\infty} s^{M} u^{M+q / 2+r} e^{-u\left(s^{2}+1\right)} d s d u \\
& =\frac{\Gamma((M+1) / 2)}{2 \Gamma(M+1+q / 2+r)} \int_{0}^{\infty} u^{q / 2+r+M / 2-1 / 2} e^{-u} d u \\
& =\frac{\Gamma((M+1) / 2) \Gamma(q / 2+r+M / 2+1 / 2)}{2 \Gamma(M+1+1 / 2)} .
\end{aligned}
$$


Putting the pieces together gives

$$
\begin{aligned}
\left.\left(B \Phi_{M+1}^{r}\right)\left(a_{0}, \ldots, a_{M}\right)\right|_{u=1} & \\
= & \frac{\eta_{M}}{2}(-1)^{M} \frac{\Gamma((M+1) / 2)}{\Gamma(q / 2+r)} \frac{\Gamma(((q-1) / 2+r)+M / 2+1)}{2 M !} \\
& \cdot \tau\left(\gamma F\left[F, a_{0}\right] \ldots\left[F, a_{M}\right]\right) .
\end{aligned}
$$

Now $\eta_{M}=\sqrt{2 i}^{P}(-1)^{M} 2^{M+1} \Gamma(M / 2+1) / \Gamma(M+1)$, and the duplication formula for the Gamma function tells us that

$$
\Gamma((M+1) / 2) \Gamma(M / 2+1) 2^{M}=\sqrt{\pi} \Gamma(M+1) .
$$

Hence

$$
\begin{aligned}
& \left.\left(B \Phi_{M+1}^{r}\right)\left(a_{0}, \ldots, a_{M}\right)\right|_{u=1} \\
& \quad=\frac{\sqrt{\pi} \sqrt{2 i}^{P} \Gamma(((q-1) / 2+r)+M / 2+1)}{\Gamma(q / 2+r) 2 \cdot M !} \tau\left(\gamma F\left[F, a_{0}\right]\left[F, a_{1}\right] \ldots\left[F, a_{M}\right]\right) .
\end{aligned}
$$

Now we use the functional equation for the Gamma function

$$
\begin{aligned}
& \Gamma(((q-1) / 2+r)+M / 2+1) \\
&= \Gamma((q-1) / 2+r) \cdot((q-1) / 2+r+M / 2) \\
& \cdot((q-1) / 2+r+M / 2-1) \ldots((q-1) / 2+r)
\end{aligned}
$$

to write this as

$$
\begin{aligned}
& \left.\left(B \Phi_{M+1}^{r}\right)\left(a_{0}, \ldots, a_{M}\right)\right|_{u=1} \\
& \quad=\frac{C_{q / 2+r} \sqrt{2 i}^{P}}{2 \cdot M !} \sum_{j=1}^{M / 2}\left((r+(q-1) / 2)^{j} \sigma_{M / 2, j} \tau\left(\gamma F\left[F, a_{0}\right]\left[F, a_{1}\right] \ldots\left[F, a_{M}\right]\right),\right.
\end{aligned}
$$

where the $\sigma_{M / 2, j}$ are elementary symmetric functions of the integers $1,2, \ldots, M / 2$ (even case) or of the half integers $1 / 2,3 / 2, \ldots, M / 2$ (odd case). Recalling that the 'constant' $C_{q / 2+r}$ has a simple pole at $r=(1-q) / 2$ with residue equal to 1 , and $\sigma_{M / 2,1}=\Gamma(M / 2+1)$ in both even and odd cases, and recalling Definition 3.2 of $\tau^{\prime}$ we see that

$$
\begin{aligned}
& \left.\frac{1}{(r-(1-q) / 2)}\left(B \Phi_{M+1}^{r}\right)\left(a_{0}, \ldots, a_{M}\right)\right|_{u=1} \\
& =\frac{\sqrt{2 i}^{P} \Gamma(M / 2+1)}{(r-(1-q) / 2) 2 \cdot M !} \tau\left(\gamma F\left[F, a_{0}\right] \ldots\left[F, a_{M}\right]\right)+\text { holo } \\
& =\frac{\sqrt{2 i}^{P} \Gamma(M / 2+1)}{M !(r-(1-q) / 2)} \tau^{\prime}\left(\gamma a_{0}\left[F, a_{1}\right] \ldots\left[F, a_{M}\right]\right)+\text { holo }
\end{aligned}
$$




$$
=\frac{1}{(r-(1-q) / 2)} \mathrm{Ch}_{F}\left(a_{0}, a_{1}, \ldots, a_{M}\right)+\text { holo, }
$$

where holo is a function holomorphic at $r=(1-q) / 2$ and on the right-hand side the Chern character appears with its $(b, B)$ normalisation.

Proof of Theorem 5.2. If we assume isolated spectral dimension we can take residues of the resolvent cocycle to obtain the residue cocycle. By Corollary 5.17, the residue cocycle is cohomologous to $B \Phi_{M+1}^{(1-q) / 2}$. Observe that it is only in Corollary 5.17 that we need to assume isolated spectral dimension. This is because by Corollary 5.25 we always have

$$
\left(b B \Psi_{u, M}^{(1-q) / 2}\right)\left(a_{0}, \ldots, a_{M}\right)=\frac{d}{d u}\left(B \Phi_{u, M+1}^{(1-q) / 2}\right)\left(a_{0}, \ldots, a_{M}\right) .
$$

Then by the computations above in the proof of Theorem 5.1, we have

$$
\left(B \Phi_{u=1, M+1}^{(1-q) / 2}\right)\left(a_{0}, \ldots, a_{M}\right) \text { is cohomologous to }\left(B \Phi_{u=0, M+1}^{(1-q) / 2}\right)\left(a_{0}, \ldots, a_{M}\right),
$$

which again by the proof of Theorem 5.1 completes the proof of the theorem.

5.7. Removing the invertibility hypothesis. Theorem 4.10 will have been proved once we show how to remove the invertibility hypothesis. We shall employ the 'double' of the spectral triple $(\mathcal{A}, \mathscr{H}, \mathscr{D})$ from Section 3.

In the double up procedure we will start with $0 \leq \mu<1$. We are interested in the relationship between $1+\mathscr{D}^{2}$ (implicitly tensored by $\operatorname{Id}_{2}$ here and below) and $1+\mathscr{D}_{\mu}^{2}$, given by

$$
1+D_{\mu}^{2}=\left(\begin{array}{cc}
1+\mu^{2}+\mathscr{D}^{2} & 0 \\
0 & 1+\mu^{2}+D^{2}
\end{array}\right) .
$$

If we scale $\mathscr{D}_{\mu}$ by $\left(1-\mu^{2}\right)^{-1 / 2}$ then we obtain

$$
\left(1+\mathscr{D}_{\mu}^{2}\right)^{-s} \longrightarrow\left(1-\mu^{2}\right)^{s}\left(1+\mathscr{D}^{2}\right)^{-s} .
$$

Let $\omega_{m, k}=a_{0}\left[\mathscr{D}, a_{1}\right]^{\left(k_{1}\right)} \ldots\left[\mathscr{D}, a_{m}\right]^{\left(k_{m}\right)}$. Then if we scale $\mathscr{D}$ by $\varepsilon, \omega_{m, k} \rightarrow$ $\varepsilon^{2|k|+m} \omega_{m, k}$. If we write $\omega_{\mu, m, k}$ for $\omega_{m, k}$ defined using $\mathscr{D}_{\mu}$, then

$$
\omega_{\mu, m, k}=\omega_{m, k}+O(\mu),
$$

where the $O(\mu)$ term is an operator of order $|k|$. In terms of the matrix representation we have

$$
\omega_{\mu, m, k}=\left(\begin{array}{cc}
\omega_{m, k}+\mu \omega_{m, k}^{\prime} & \mu \omega_{m, k}^{\prime \prime} \\
0 & 0
\end{array}\right)
$$


Now take $\omega_{\mu, m, k}\left(1+\mathscr{D}_{\mu}^{2}\right)^{-r / 2-|k|-m / 2+1 / 2-p / 2}$ and scale $\mathscr{D}_{\mu}$ by $\left(1-\mu^{2}\right)^{-1 / 2}$. We obtain

$$
\begin{gathered}
\left(1-\mu^{2}\right)^{(q-1) / 2+r} \omega_{m, k}\left(1+D^{2}\right)^{-r-|k|-m / 2-(q-1) / 2} \\
+O(\mu)\left(1+D^{2}\right)^{-r-|k|-m / 2-(q-1) / 2},
\end{gathered}
$$

where again the $O(\mu)$ term is an order $|k|$ operator.

Let us write $\zeta_{m, \mu}^{r}$ for the sum of zeta functions we obtain by performing the pseudodifferential calculus on the resolvent cocycle and discarding the holomorphic remainder. Then, modulo coboundaries and functions holomorphic at $r=(1-q) / 2$,

$$
\begin{aligned}
\frac{1}{(r-(1-q) / 2)} \operatorname{Ch}_{F_{\mu}} & =\left(\phi_{m, \mu}^{r}\right)_{m=P}^{M} \\
& =\left(\zeta_{m, \mu}^{r}\right)_{m=P}^{M} \\
& =\left(1-\mu^{2}\right)^{r-(1-q) / 2}\left(\zeta_{m, 0}^{r}\right)_{m=P}^{M}+O(\mu) \\
& =\left(1-\mu^{2}\right)^{r-(1-q) / 2}\left(\phi_{m}^{r}\right)_{m=P}^{M}+O(\mu) \\
& =\left(\phi_{m}^{r}\right)_{m=P}^{M}+O(\mu),
\end{aligned}
$$

where each $O(\mu)$ is a cocycle with the same regularity properties as the resolvent cocycle and which is zero at $\mu=0$.

Evaluating both sides of this equation on a $b, B$-cycle yields functions defined and holomorphic in some half-plane, and the two sides differ by functions holomorphic in a half-plane containing $r=(1-q) / 2$. The left-hand side yields a function independent of $\mu$ by Proposition 3.5, and so either the $O(\mu)$ contributions are coboundaries (and so vanish when evaluated on a cycle) or they are holomorphic at $r=(1-q) / 2$. In either case we find that modulo cochains yielding functions holomorphic at $r=(1-q) / 2$,

$$
\left(\phi_{m}^{r}\right)_{m=P}^{M} \text { is cohomologous to } \frac{1}{(r-(1-q) / 2)} \mathrm{Ch}_{F_{\mu}} .
$$

Taking residues, in the case that $(\mathcal{A}, \mathscr{H}, \mathscr{D})$ has isolated spectral dimension, leads to the analogous result for the residue cocycle:

$$
\left(\phi_{m}\right)_{m=P}^{M} \text { is cohomologous to } \mathrm{Ch}_{F_{\mu}} \text {. }
$$

Since $\operatorname{Ch}(\mathscr{A}, \mathscr{H}, \mathscr{D})$ is $\mathrm{Ch}_{F_{\mu}}$ which is $\operatorname{Ch}\left(\mathcal{A}, \mathscr{H}^{2}, \mathscr{D}_{\mu}\right)$ for any positive $\mu$, we are done.

Theorem 5.26. If $(\mathcal{A}, \mathcal{H}, \mathcal{D})$ is a $Q C^{\infty}$ finitely summable spectral triple with spectral dimension $q \geq 1$, then the resolvent cocycle is cohomologous to

$$
\frac{1}{(r-(1-q) / 2)} \mathrm{Ch}_{F_{\mu}}
$$


modulo cochains with values in the functions holomorphic at $r=(1-q) / 2$. If $(\mathcal{A}, \mathscr{H}, \mathcal{D})$ also has isolated spectral dimension, then the cyclic cohomology class of the residue cocycle coincides with the class of the Chern character of $\left(\mathscr{H}, F=\mathscr{D}\left(1+\mathscr{D}^{2}\right)^{-1 / 2}\right)$.

\section{Some corollaries and the connection with Higson's cocycle}

The transgression cocycle of the previous section allows us to prove two interesting corollaries.

Corollary 6.1. For any $(b, B)$ cycle $\left(c_{m}\right)_{m=P}^{K}$ of the same parity as $(\mathcal{A}, \mathscr{H}, \mathcal{D})$, the function

$$
\sum_{m=P}^{M} \phi_{m}^{r}\left(c_{m}\right)
$$

has an analytic continuation to a deleted neighbourhood of $r=(1-q) / 2$ with at worst a simple pole at $r=(1-q) / 2$.

Proof. The cocycle $\left(\phi_{m}^{r}\right)$ differs from $(1 /(r-(1-q) / 2)) \mathrm{Ch}_{F}$ by coboundaries and functions holomorphic at $r=(1-q) / 2$.

Corollary 6.2. For any Hochschild $M$-cycle $c_{M}$ the function

$$
\phi_{M}^{r}\left(c_{M}\right)
$$

has an analytic continuation to a deleted neighbourhood of $r=(1-q) / 2$ with at worst a simple pole at $r=(1-q) / 2$. If $M$ is even (resp. odd) and $[q]$ is odd (resp. even) the residue at $r=(1-q) / 2$ vanishes.

Proof. We have the formula

$$
b \Phi_{M-1}^{r}+B \Phi_{M+1}^{r}=(r-(1-q) / 2) \phi_{M}^{r} .
$$

If $c_{M}=\sum_{i} a_{0}^{i} \otimes a_{1}^{i} \otimes \cdots \otimes a_{M}^{i}$ is a Hochschild cycle, we have $b \Phi_{M-1}^{r}\left(c_{M}\right)=0$, so

$$
\frac{1}{r-(1-q) / 2} B \Phi_{M+1}^{r}\left(c_{M}\right)=\phi_{M}^{r}\left(c_{M}\right) .
$$

Since there exists $\delta>0$ with $B \Phi_{M+1}^{r}\left(c_{M}\right)$ holomorphic for $\operatorname{Re}(r)>(1-q) / 2-\delta$, we see that $\phi_{M}^{r}\left(c_{M}\right)$ meromorphically continues to this region with only a simple pole at $r=(1-q) / 2$. The region $\operatorname{Re}(r)>(1-M) / 2$ where $\phi_{M}^{r}\left(a_{0}, \ldots, a_{M}\right)$ is holomorphic is $\operatorname{Re}(r)>-[q / 2]$. For $[q]=2 n, q=2 n+\kappa, 0 \leq \kappa<1$ and

$$
\frac{1-q}{2}=-n+\frac{1-\kappa}{2}>-[q / 2]=-n \text {. }
$$


Similar comments apply when $M$ is even and $[q]$ is odd.

Higson has a cocycle which is evidently similar to our resolvent cocycle. An essential difference is that from Higson's cocycle, one derives the unrenormalised local index theorem. We show here that our resolvent cocycle naturally gives rise to a 'renormalised' version of Higson's cocycle.

We take our resolvent cocycle, perform the pseudodifferential expansion, the Cauchy integral and the $s$-integral. This gives (modulo functions holomorphic at $r=(1-q) / 2)$

$$
\begin{aligned}
\phi_{m}^{r}\left(a_{0}, \ldots, a_{m}\right) \\
=\sum_{|k|=0}^{2 N-m-P} C(k)(-1)^{m+|k|} \sqrt{\pi}(-1)^{P} \sqrt{2 i} \frac{P(|k|+(m-1) / 2+q / 2+r)}{\Gamma(1+|k|+m) \Gamma(q / 2+r)} \\
\quad \cdot \tau\left(\gamma a_{0}\left[\mathcal{D}, a_{1}\right]^{\left(k_{1}\right)} \ldots\left[\mathcal{D}, a_{m}\right]^{\left(k_{m}\right)}\left(1+\mathcal{D}^{2}\right)^{-q / 2-r-|k|-(m-1) / 2}\right) .
\end{aligned}
$$

We then put back the Cauchy integral using

$$
\begin{gathered}
\tau\left(\gamma a_{0}\left[\mathscr{D}, a_{1}\right]^{\left(k_{1}\right)} \ldots\left[\mathcal{D}, a_{m}\right]^{\left(k_{m}\right)}\left(1+\mathscr{D}^{2}\right)^{-q / 2-r-|k|-(m-1) / 2}\right) \\
=(-1)^{|k|+m} \frac{\Gamma(1+|k|+m) \Gamma(q / 2+r-(m+1) / 2)}{\Gamma(q / 2+r+|k|+(m-1) / 2)} \\
\cdot \tau\left(\frac{1}{2 \pi i} \int_{l} \lambda^{-q / 2-r+(m+1) / 2} a_{0}\left[\mathscr{D}, a_{1}\right]^{\left(k_{1}\right)} \ldots\right. \\
\left.\ldots\left[\mathscr{D}, a_{m}\right]^{\left(k_{m}\right)}\left(\lambda-\left(1+\mathscr{D}^{2}\right)\right)^{-|k|-m-1} d \lambda\right)
\end{gathered}
$$

and undo the pseudodifferential expansion. By our previous estimates, these operations affect our function-valued cocycle only by functions holomorphic at the critical point $r=(1-q) / 2$. We obtain the following equality modulo functions holomorphic at $r=(1-q) / 2$ :

$$
\begin{aligned}
& \phi_{m}^{r}\left(a_{0}, \ldots, a_{m}\right) \\
& =(-1)^{P} \sqrt{2 i}^{P} \frac{\sqrt{\pi} \Gamma(q / 2+r-(m+1) / 2)}{\Gamma(q / 2+r)} \\
& \quad \cdot \tau\left(\frac{1}{2 \pi i} \int_{l} \lambda^{-q / 2-r+(m+1) / 2} a_{0} R_{0}(\lambda)\left[\mathcal{D}, a_{1}\right] R_{0}(\lambda) \ldots\left[\mathcal{D}, a_{m}\right] R_{0}(\lambda) d \lambda\right) .
\end{aligned}
$$

We call this new cocycle the reduced resolvent cocycle and denote its components by $\psi_{m}^{r}$ so that the above equality becomes

$$
\phi_{m}^{r}\left(a_{0}, \ldots, a_{m}\right)=\psi_{m}^{r}\left(a_{0}, \ldots, a_{m}\right)
$$


modulo functions holomorphic at $r=(1-q) / 2$. The integral defining $\psi_{m}^{r}$ exists for $\operatorname{Re}(r)>(1-m) / 2$ by our previous estimates. The argument of the coefficient

$$
\Gamma(q / 2+r-(m+1) / 2)
$$

has positive real part when $\operatorname{Re}(r)>m / 2+(1-q) / 2$ and can be meromorphically continued.

To compare the reduced resolvent cocycle with Higson's improper cocycle, we write $z=r-(1-q) / 2$. Then, writing $\eta_{m}^{z}$ for the components of Higson's improper cocycle we have

$$
\psi_{m}^{r}\left(a_{0}, \ldots, a_{m}\right)=\frac{\sqrt{\pi}}{\Gamma(z+1 / 2)} \eta_{m}^{z} .
$$

This gives a 'renormalised' version of Higson's cocycle in the sense that starting with the reduced resolvent cocycle, one arrives at the renormalised local index theorem, whereas Higson's original cocycle leads to the unrenormalised theorem.

\section{References}

[BCPRSW] M.-T. Benameur, A. L. Carey, J. Phillips, A. Rennie, F. A. Sukochev, and K. P. Wojciechowski, An analytic approach to spectral flow in von Neumann algebras. In Analysis, geometry and topology of elliptic operators, World Scientific, Singapore 2006, 297-352. Zbl 1119.58016 MR 2246773

[BeF] M.-T. Benameur and T. Fack, Type II non-commutative geometry. I. Dixmier trace in von Neumann algebras. Adv. Math. 199 (2006), 29-87. Zbl 1092.46050 MR 2186918

[B1] M. Breuer, Fredholm theories in von Neumann algebras. I. Math. Ann. 178 (1968), 243-254. Zbl 0162.18701 MR 0234294

[B2] M. Breuer, Fredholm theories in von Neumann algebras. II. Math. Ann. 180 (1969), 313-325. Zbl 0175.44102 MR 0264407

[CP0] A. L. Carey and J. Phillips, Algebras almost commuting with Clifford algebras in a II $\infty$ factor. K-Theory 4 (1991), 445-478. Zbl 0732.46039 MR 1116928

[CP1] A. Carey and J. Phillips, Unbounded Fredholm modules and spectral flow. Canad. J. Math. 50 (1998), 673-718. Zbl 0915.46063 MR 1638603

[CP2] A. Carey and J. Phillips, Spectral flow in Fredholm modules, eta invariants and the JLO cocycle. K-Theory 31 (2004), 135-194. Zbl 1051.19004 MR 2053481

[CPS1] A. L. Carey, J. Phillips, and F. A. Sukochev, On unbounded $p$-summable Fredholm modules. Adv. Math. 151 (2000), 140-163. Zbl 0960.46039 MR 1758245

[CPS2] A. Carey, J. Phillips, and F. Sukochev, Spectral flow and Dixmier traces. Adv. Math. 173 (2003), 68-113. Zbl 1015.19003 MR 1954456 
[CPRS1] A. L. Carey, J. Phillips, A. Rennie, and F. A. Sukochev, The Hochschild class of the Chern character for semifinite spectral triples. J. Funct. Anal. 213 (2004), 111-153. Zbl 1058.19002 MR 2069783

[CPRS2] A. L. Carey, J. Phillips, A. Rennie, and F. A. Sukochev, The local index formula in semifinite von Neumann algebras I: Spectral flow. Adv. Math. 202 (2006), 451-516. Zbl 1118.46060 MR 2222358

[CPRS3] A. L. Carey, J. Phillips, A. Rennie, and F. A. Sukochev, The local index formula in semifinite von Neumann algebras II: The even case. Adv. Math. 202 (2006), 517-554. Zbl 1101.46045 MR 2222359

[CDSS] L. A. Coburn, R. G. Douglas, D. G. Schaeffer, and I. M. Singer, $C^{*}$-algebras of operators on a half-space II: Index theory. Inst. Hautes Études Sci. Publ. Math. 40 (1971), 69-79. Zbl 0241.47027 MR 0358418

[Co1] A. Connes, Non-commutative differential geometry. Inst. Hautes Études Sci. Publ. Math. 62 (1985), 257-360. Zbl 0592.46056 MR 823176

[Co4] A. Connes, Noncommutative geometry. Academic Press, San Diego 1994. Zbl 0818.46076 MR 1303779

[Co5] A. Connes, Geometry from the spectral point of view. Lett. Math. Phys. 34 (1995), 203-238. Zbl 1042.46515 MR 1345552

[CoM] A. Connes and H. Moscovici, The local index formula in noncommutative geometry. Geom. Funct. Anal. 5 (1995), 174-243. Zbl 0960.46048 MR 1334867

[CM1] A. Connes and H. Moscovici, Transgression du caractère de Chern et cohomologie cyclique. C. R. Acad. Sci. Paris Sér. I Math. 303 (1986), 913-918. Zbl 0617.46075 MR 873393

[Dix] J. Dixmier, Les algèbres d'opérateurs dans l'espace hilbertien (Algèbres de von Neumann). 2nd. ed., Cahiers Scientifiques, Fasc. XXV, Gauthier-Villars Éditeur, Paris 1969. Zbl 0175.43801 MR 0352996

[FK] T. Fack and H. Kosaki, Generalized $s$-numbers of $\tau$-measurable operators. Pacific J. Math. 123 (1986), 269-300. Zbl 0617.46063 MR 840845

[G] E. Getzler, Cyclic homology and the Atiyah-Patodi-Singer index theorem. In Index theory and operator algebras (Boulder, CO, 1991), Contemp. Math. 148, Amer. Math. Soc., Providence, RI, 1993, 19-45. Zbl 0798.46049 MR 1228498

[GVF] J. M. Gracia-Bondía, J. C. Várilly, and H. Figueroa, Elements of noncommutative geometry. Birkhäuser Adv. Texts, Birkhäuser, Boston 2001. Zbl 0958.46039 MR 1789831

$[\mathrm{H}] \quad$ N. Higson, The local index formula in noncommutative geometry. In Contemporary developments in algebraic $K$-theory, ICTP Lecture Notes 15 , Abdus Salam Int. Cent. Theoret. Phys., Trieste 2003, 447-536. Zbl 02143530 MR 2175637

[JLO] A. Jaffe, A. Lesniewski, and K. Osterwalder, Quantum $K$-theory I. The Chern character. Comm. Math. Phys. 118 (1988), 1-14. Zbl 0656.58048 MR 954672

[K] G. G. Kasparov, The operator $K$-functor and extensions of $C^{*}$-algebras. $I z v$. Akad. Nauk SSSR Ser. Mat. 44 (1980), 571-636; English transl. Math. USSR-Izv. 16 (1981), 513-572. Zbl 0464.46054 MR 582160 
[L] M. Lesch, On the index of the infinitesimal generator of a flow. J. Operator Theory 26 (1991), 73-92. Zbl 0784.46041 MR 1214921

[Lo] J.-L. Loday, Cyclic homology. Grundlehren Math. Wiss. 301, 2nd ed., SpringerVerlag, Berlin 1998. Zbl 0885.18007 MR 1600246

[M] V. Mathai, Spectral flow, eta invariants, and von Neumann algebras. J. Funct. Anal. 109 (1992), 442-456. Zbl 0783.57015 MR 1186326

[PR] J. Phillips and I. Raeburn, An index theorem for Toeplitz operators with noncommutative symbol space. J. Funct. Anal. 120 (1994), 239-263. Zbl 0815.47033 MR 1266310

[R] A. Rennie, Smoothness and locality for nonunital spectral triples. $K$-Theory 28 (2003), 127-165. Zbl 1027.46088 MR 1995874

[Ru] W. Rudin, Principles of mathematical analysis, 3rd ed. McGraw-Hill, New York 1976. Zbl 0346.26002 MR 0385023

[Sh] M. A. Shubin, Pseudodifferential almost-periodic operators and von Neumann algebras. Trudy Moskov. Mat. Obshch. 35 (1976), 103-164; English transl. Trans. Moscow Math. Soc. 1979 (1979), no.1, 103-166. Zbl 0405.47035 MR 0636409

Received September 19, 2006

A. L. Carey, Mathematical Sciences Institute, Australian National University, Canberra, ACT. 0200, Australia

E-mail: acarey@maths.anu.edu.au

J. Phillips, Department of Mathematics and Statistics, University of Victoria, Victoria, B.C. V8W 3P4, Canada

E-mail: phillips@math.uvic.ca

A. Rennie, Institute for Mathematical Sciences, University of Copenhagen, Universitetsparken 5, 2100 Copenhagen, Denmark

E-mail: rennie@math.ku.dk

F. A. Sukochev, School of Informatics and Engineering, Flinders University, Bedford Park, SA 5042, Australia

E-mail: sukochev@infoeng.flinders.edu.au 